\title{
SUR L'UNIFORMISATION DES FONCTIONS ANALYTIQUES
}

PAR

\author{
H. POINCAR'É \\ à PARIS,
}

\section{$\S$ I. Introduction.}

Dans un mémoire intitulé «Sur un théorème de la théorie générale des fonctions» (Bulletin de la Société Mathématique de France, tome II, I883), j'ai démontré que plusieurs fonctions analytiques d'une même variable indépendante peuvent toujours être égalées à des fonctions uniformes d'une même variable.

La question a été reprise par M. Osgood, puis par M. Johansson: Ueber die Uniformisirung RIEMANN'scher Flächen mit endlicher Anzahl Windungspunkte (Acta Societatis Scientiarum Fennicæ, tome 33, $\mathrm{N}^{\circ} 7$ ) et enfin dans un intéressant travail de M. BrodíN, Bemerkungen über die Uniformisirung analytischer Funktionen (Lund, Berlingsche Buchdruckerei I905) que nous aurons l'occasion de citer plus loin.

Mon premier mémoire laissait subsister plusieurs questions non résolues. $1^{\circ}$ Pour tout point de la surface de Ribmans, pour lequel les fonctions données existent, les fonctions uniformisantes se comportent régulièrement. Il y a exception pour trois de ces points qui constituent ce que M. Hinbert appelle des Ausnahmsstellen. Cette difficulté a été signalée de nouveau par M. HruBRrT dans sa communication au Congrès des Mathematiciens de Paris en I900.

Elle tient à ce qu'on introduit une fonction auxiliaire, inverse d'une fonction fuchsienne et que pour ces trois points, cette fonction auxiliaire n'existe pas. Il est possible de l'écarter, et cela de deux manières; d'abord en remplaçant la fonction auxiliaire par une autre, également inverse d'une fonction fuchsienne et qui ne présente pas le même inconvenient; c'est ce que je fais plus loin au $\S 4$; l'autre manière consiste à se passer complètement de fonction auxiliaire, c'est ce que je fais aux $\$$ I3 et I4.

$2^{\circ}$. Mes procédés permettaient bien de démontrer que l'on pouvait faire la représentation conforme de ma surface de RIEMANN sur une aire intérieure à un cercle; mais on ne voyait pas que ce fût possible sur un cercle; M. Osaoon a écarté cettè difficulté; nous aurons néanmoins à y revenir au $\$ 8$.

Aeta mathematica. 31. Imprimé le 19 mars 1907. 
H. Poincaré.

$3^{\circ}$. On n'était pas certain que cette façon d'uniformiser les fonctions fût la plus simple de toutes; on était même certain du contraire par l'exemple des fonctions fuchsiennes. L'introduction de la fonction auxiliaire, arbitraire dans une large mesure, donnait à la solution un caractère artificiel dont il convenait de se débarrasser, et pour cela il fallait démontrer que toute surface de Rremans, simplement connexe, est représentable soit sur un cercle, soit sur une sphère pointée (ou ce qui revient au même sur le plan tout entier).

Le problème n'est autre chose que le problème de Dirichlet appliqué à une surface de Rremans à une infinité de feuillets. Malheureusement les procédés ordinaires ne sont pas toujours applicables pour deux raisons: $I^{0}$ parce que le domaine auquel on veut l'appliquer n'est pas une aire plane à un seul feuillet, mais est formé de plusieurs feuillets superposés; $2^{\circ}$ parce que les frontières de ce domaine ne sont pas nettement délimitées et que souvent même on ne peut pas les atteindre, mais s'en approcher pour ainsi dire par une suite indéfinie d'approximations. De là la nécessité de faire grande attention aux détails de la démonstration, ce qui entraîne une assez grande prolixité.

Dans le $\S 2$, je précise la notion de la surface de Rremann qu'il va s'agir «d'uniformiser». Je la considère comme un domaine formé d'une suite d'éléments, imbriqués les uns dans les autres et correspondant aux divers éléments d'une fonction analytique au sens Werrrstrassier du mot. Je montre comment on peut généraliser cette notion et la rendre plus souple, principalement par l'introduction des éléments algébriques qui permettent de traiter les points de ramification algébrique de la surface de RIEManN comme faisant partie de cette surface. J'étndie les relations mutuelles des diverses surfaces de RiEmann correspondant aux divers systèmes de fonctions analytiques.

Dans le $\$ 3$, je forme la fonction de Grewn relative à ma surface de RIEMANN; et je la représente par une serie qui converge quand cette fonction existe. Je modifie la démonstration donnée dans mon mémoire primitif en la rattachant à la méthode du balayage et je la simplifie par l'application du théorème de HARNACK.

Dans la $\$ 4$, j'introduis la fonction auxiliaire majorante nécessaire pour assurer la convergence de la serie; je montre que cette fonction peut être choisie de façon à éviter les Ausnahmsstellen.

Dans le \& 5 , je déduis de la fonction de Green, la fonction analytique $z$ qui doit assurer la représentation conforme de la surface de RiEMaNN sur un cercle.

Dans le $\$ 6$, je généralise la méthode du balayage de façon à y faire rentrer celle que j'avais employée dans mon mémoire primitif.

Dans le $\$ 7$, je compare les diverses fonctions $z$ qu'on peut déduire d'une 
même surface de RiemanN, et je montre qu'elles sont liées par des relations linéaires; je montre ensuite que chacune d'elles ne peut prendre plus d'une fois la même valeur.

Il s'agit ensuite de montrer que la surface de RiEmans peut être représentée sur un cercle; c'est ce qu'a fait Osqood, je reproduis sa démonstration dans le $\$ 8$, mais en la préséntant de manière à faire voir que c'est bien par la fonction $z$ que se fait la réprésentation. Je donne ensuite du même théorème une seconde démonstration.

Dans les $\S 9$, Io et II, j'étudie les propriétés des fonctions uniformisantes; je montre leur analogie avec les fonctions fuchsiennes, leurs relations avec un groupe analogue aux groupes fuchsiens et la possibilité de les représenter par des séries analogues aux séries thétafuchsiennes.

A la fin du mémoire, je cherche à me passer de la fonction auxiliaire majorante du $\$ 4$. Je considère un domaine $D$ (ou surface de RiEmans) simplement connexe, mais d'ailleurs quelconque. J'en enlève une aire simplement connexe, et il me reste un domaine $D_{1}$ doublement connexe. Au $\$ 12$ je forme la fonction de Green relative à ce domaine et je montre qu'elle existe toujours. Je suis amené ensuite à distinguer deux cas.

Le premier cas est examiné aux \$ I3 et 14; je montre que dans ce cas le domaine $D_{1}$ est représentable sur une couronne circulaire et le domaine $D$ sur un cercle.

Dans le second cas que j'étudie au $\$ 15$, le domaine $D_{1}$ est représentable sur un cercle, et le domaine $D$ sur une sphère.

Les critères qui permettent de discerner entre les deux cas peuvent se présenter sous des formes très différentes; on comparera celles que j'ai données au $\S \mathrm{I}$, à la fin du $\S \mathrm{I} 2$, au $\S \mathrm{I} 3$, au $\S \mathrm{I} 5$; et on les rapprochera des critère proposés par M. BRODÉN.

Le théorème final ( $\$ 14$ et 15 ) permettrait de démontrer l'existence des fonctions fuchsiennes d'un type donné, sans avoir recours ni à la méthode dite de continuité, ni à l'équation $\Delta u=e^{u}$.

\section{$\S 2$. Définition des domaines $D$.}

Représentons-nous une fonction analytique comme le faisait WeTERSTRASs. Un élément de fonction, c'est une série de puissances $R$ convergente à l'intérieur d'un cercle $C$. Deux éléments sont contigus quand leurs cercles de convergence $C$ et $C^{\prime}$ ont une partie commune, et quand en tout point de cette partie commune, les deux séries $R$ et $R^{\prime}$ correspondant à ces deux éléments ont même somme. 
H. Poincaré.

Une fonction analytique sera alors constituée par un ensemble dénombrable d'éléments de fonction, tels que l'on peut passer de l'un quelconque de ces éléments $E$ à un autre quelconque $E^{\prime}$ par une chaîne formée par un nombre fini d'éléments, le premier élément de la chaîne étant $E$ et le dernier $E^{\prime}$, et chaque élément de la chaîne étant contigu au suivant et au précédent. Pourquoi cet ensemble doit-il être dénombrable, c'est ce que j'ai expliqué au tome 2 des Rendiconti del Circolo Matematico di Palermo.

Cela posé, voyons ce que nous devons entendre par le domaine $\boldsymbol{D}$ d'une fonction analytique $\boldsymbol{F}^{\prime}$ quelconque. C'est l'ensemble des cercles de convergence $\boldsymbol{C}$ relatifs aux différents éléments de $\boldsymbol{F}$, mais avec la convention suivante: pour que deux points du domaine soient regardés comme identiques, il ne suffit pas qu'ils aient mêmes coordonnées. Soient $E$ et $E^{\prime}$ deux éléments de la fonction, $C$ et $C^{\prime}$ les cercles de convergence correspondants; $R$ et $R^{\prime}$ les deux séries correspondantes, soient $M$ et $M^{\prime}$ deux points considérés comme appartenant le $\mathrm{I}^{\mathrm{er}} \grave{\mathrm{a}} C$, le $2^{\mathrm{d}} C^{\prime}$, et ayant d'ailleurs mêmes coordonnées; les deux points coincident donc au point de vue géométrique, mais il ne s'ensuit pas qu'ils soient identiques à notre nouveau point de vue; de l'un de ces deux points comme centre, je décris un cercle $K$ assez petit pour être contenu tout entier tant dans $C$ que dans $C^{\prime}$; si les deux séries $R$ et $R^{\prime}$ ont même somme à l'intérieur de $K$ les deux points $M$ et $M^{\prime}$ seront regardés comme identiques, sinon non. Ainsi notre domaine sera un plan à plusieurs feuillets et en général à une infinité de feuillets; ce sera une surface de Riemann analogue à la surface $\boldsymbol{S}$ envisagée dans le mémoire cité.

On pourra faire une convention différente; on pourra convenir que l'égalité des séries $R$ et $R^{\prime}$ reste une condition nécessaire pour que les deux points $M$ et $M^{\prime}$ soient identiques, mais que cette condition ne soit plus suffisante. Nous pourrons même convenir de regarder deux éléments de la fonction $F$ comme non identiques, bien que les cercles $C$ et $C^{\prime}$ correspondants soient les mêmes de même que les deux séries $R$ et $R^{\prime}$. Si par exemple on a $F=\sqrt{z}$, et qu'on parte d'un élément quelconque de cette fonction, l'élément sur lequel on retombera après avoir tourné deux fois autour du point $z=0$ sera généralement regardé comme identique à l'élément initial. Mais nous pourrons également convenir de le regarder comme différent.

On définira ainsi un autre domaine $D^{\prime}$, nous pourrons appeler $D$ le drmaine principal de la fonction $F^{\prime}$; les divers domaines $D^{\prime}$ seront les domaines secondaires de cette même fonction. Alors à chaque point d'un domaine secondaire $D^{\prime}$ correspondra un point et un seul du domaine principal $D$, mais à un point de $D$ pourront correspondre plusieurs points de $D^{\prime}$ et en effet pour que deux points de $D^{\prime}$ soient identiques il faut, mais il ne suffit pas que les deux points correspondants de $D$ soient identiques. 
Pour définir un domaine secondaire $D^{\prime}$, nous devons, d'après ce qui précóde, énoncer les conditions nécessaires et suffisantes pour que deux de ses points $M$ et $M^{\prime}$ doivent être regardés comme identiques. Le choix de ces conditions reste arbitraire dans une très large mesure, il est cependant soumis aux restrictions suivantes. Soient deux points $M$ et $M^{\prime}$, ayant mêmes coordonnées et appartenant respectivement à deux éléments de fonctions; soient $C$ et $C^{\prime}$ les deux cercles, $R$ et $R^{\prime}$ les deux séries correspondant à ces deux éléments. Il s'agit de savoir à quelles conditions les deux points $M$ et $M^{\prime}$ seront identiques.

$I^{\circ}$. La condition que $R=R^{\prime}$ dans le voisinage des points $M$ et $M^{\prime}$ n'est plus suffisante, mais reste nécessaire.

$2^{\circ}$. Si les points $M$ et $M^{\prime}$ ont mêmes coordonnées il y aura une infinité de couples de points $N$ et $N^{\prime}$ appartenant respectivement à $C$ et à $C^{\prime}$ et qui auront mêmes coordonnées. Si $M$ et $M^{\prime}$ sont identiques, il devra en être de même pour tous les couples de points tels que $N$ et $N^{\prime}$.

$3^{\circ}$. Deux points identiques à un même troisième sont identiques entre eux.

$4^{\circ}$. Si nous considérons deux éléments quelconques de la fonction $F$, et que les cercles correspondants soient $C$ et $C^{\prime}$; il faudra que l'on puisse passer de l'un à autre par une chaîne d'un nombre fini d'éléments, dont les cercles soient respectivement $C, C_{1}, C_{2}, \ldots, C_{n}, C^{\prime}$; et de telle façon que si l'on considère deux cercles consécutifs de cette chaîne, il y ait un point intérieur à l'un qui soit identique à un point interieur à l'autre.

On voit aussi la possibilité de définir un domaine, indépendamment de toute fonction $F$. Il suffit de se représenter un ensemble dénombrable de cercles $C_{1}, C_{2}, \ldots$ en convenant que deux points appartenant à deux de ces cercles peuvent ne pas être identiques bien qu'ayant mêmes coordonnées, ou même que deux de ces cercles peuvent coïncider sans être regardés comme identiques. Il faut alors pour achever de définir le domaine, énoncer les conditions nécessaires et suffisantes pour que deux points $M$ et $M^{\prime}$ soient identiques; ces conditions devront rester soumises aux restrictions énoncées plus haut; la Ire ici n'a plus de sens, mais les 3 autres doivent subsister.

Les cercles $C_{1}, C_{2}, \ldots$, sont les éléments du domaine, et deux de ces éléments sont dits contigus quand les deux cercles ont une partie commune et que dans cette partie commune, les points correspondants sont identiques. Si alors $D$ est le domaine principal ou un domaine secondaire d'une fonction $F$, et si deux éléments de $D$ sont contigus, il en est de même des deux éléments correspondants de $F$; mais la réciproque, vraie pour le domaine principal, n'est pas vraie pour un domaine secondaire.

Observons maintenant qu'étant donnée une fonction analytique, on peut 
construire pour cette fonction plusieurs domaines principaux. Il y a en effet une infinité de manières de décomposer cette fonction en un ensemble dénombrable d'éléments de fonctions. Soit par exemple la fonction $V_{z}$, nous pouvons prendre une infinité d'éléments, dont les cercles de convergence iront tous passer par l'origine; mais les centres de ces cercles, qui doivent former un ensemble dénombrable pourront être choisis arbitrairement dans une certaine mesure. Il suffit de s'arranger de façon que tout point du plan soit intérieur au moins à l'un de ces cercles.

Comme nous pouvons choisir d'une infinité de manières ces cercles de convergence, il y a, si nous conservons la définition qui précéde, une infinité de domaines qui peuvent être regardés comme le domaine principal d'une même fonction analytique. Tous ces domaines devront cependant être regardés comme équivalents. Cette notion de l'équivalence de deux domaines peut aussi se définir sans faire intervenir la fonction $F$. Deux domaines seront équivalents quand on pourra établir entre les points de l'un et ceux de l'autre une correspondance biunivoque, de telle façon qu'à tout point de l'un corresponde un point de l'autre et un seul, à deux points identiques de l'un deux points identiques de l'autre, à deux points non identiques de l'un, deux points non identiques de l'autre; et à deux points infiniment voisins de l'un, deux points infiniment voisins de l'autre.

Un domaine $D$ est contenu dans un domaine $D_{1}$ quand à tout point de $D$ correspond un point de $D_{1}$ et un seul; quand à un point de $D_{1}$ ne peut correspondre qu'ou bien un seul point de $D$, ou bien aucun point de $D$; quand enfin deux points de l'un sont identiques, non identiques ou infiniment voisins si les deux points correspondants de l'autre sont identiques, non identiques ou infiniment voisins.

Un domaine $D$ sera multiple d'un domaine $D_{1}$, quand à tout point de $D$ correspondra un point de $D_{1}$ et un seul, tandis qu'à tout point de $D_{1}$ correspondent plusieurs points de $D$ ou même une infinité; de sorte que à deux points identiques de $D$ correspondent deux points identiques de $D_{1}$, tandisque la réciproque peut ne pas être vraie; de sorte enfin qu'à deux points infiniment voìsins de $D$ correspondent deux points infiniment voisins de $D_{1}$. Complétons toutes ces définitions (domaines équivalents, contenus ou multiples) en disant que deux points correspondants doivent avoir mêmes coordonnées. Il est clair alors que si $\boldsymbol{D}$ est le domaine principal et $\boldsymbol{D}^{\prime}$ un domaine secondaire d'une fonction analytique $\boldsymbol{F}, \boldsymbol{D}^{\prime}$ est multiple de $\boldsymbol{D}$.

Quelques remarques encore. Nous avons donné plus haut la définition d'une fonction analytique $F$ et de son domaine principal $D$; mais si l'on s'en tient à cette définition, il est permis de supposer que la fonction pourrait encore être 
prolongée analytiquement au delà de son domaine $D$, et que la frontière de ce domaine n'est pas une limite naturelle, mais une limite artificielle. C'est ainsi qu'un élément unique de fonction, une seule série avec son cercle de convergence satisferait à notre définition. Dans ce cas nous dirons que la fonction $F$ est incomplète. Elle sera au contraire complète, si elle ne peut pas être prolongée, analytiquement en dehors de son domaine. Si on a une fonction incomplète, la fonction obtenue en la prolongeant analytiquement de toutes les manières possibles sera la fonction complétée. Il résulte de là que le domaine d'une fonction incomplète est contenu dans le domaine de sa fonction complétée.

Nous pouvons étendre la notion d'élément; et par exemple admettre des éléments infinis de fonction formés par la partie du plan extérieure à un cercle $C$, de centre $a$, et par une série procédant suivant les puissances négatives de $x-a$ et convergeant à l'extérieur du cercle $C$.

Ce n'est pas tout. Nous regardons en général la circonférence d'un de nos cercles comme ne faisant pas partie de l'élément correspondant. Si alors la fonctions $\boldsymbol{F}$ possède des pôles, d'après la définition précédente, ces pôles ne feraient pas partie de son domaine, puisqu'ils ne seraient à l'intérieur d'aucun de nos cercles de convergence mais seulement sur la circonférence, Mais nous pouvons envisager outre les éléments de fonction considérés jusqu'ici et que nous appellerons éléments holomorphes, et qui sont formés d'une série de puissances et de son cercle de convergence, nous pouvons, dis-je, envisager ce que nous apellerons des éléments polaires et qui seront formés d'un cercle de convergence et du quotient de deux séries de puissances. Si nous introduisons ces éléments, nous pouvons étendre le domaine $D$ de la fonction $F$ de façon que les pôles en fassent partie.

Nous pouvons même envisager une $3^{\mathrm{a}}$ sorte d'éléments, les éléments algébriques formés d'un cercle de convergence et d'une série de puissances fractionnaires, cela pourrait nous permettre d'étendre le domaine d'une fonction de façon que ses points de ramification algébriques en fissent partie. Ce seraient seulement des points singuliers de ce domaine, et les éléments correspondants seraient singuliers même au point de vue géométrique (c'est à dire en faisant abstraction de la fonction $\boldsymbol{F}$ qui leur a donné naissance) car un pareil élément ne serait pas un cercle simple, mais un cercle multiple, $n^{\text {ple }}$ si $n$ est le dénominateur des exposants fractionnaires de notre série; de telle façon qu'il y aurait à l'intérieur de ce cercle, $n$ points, qui auraient mêmes coordonnées et qui devraient néanmoins être regardés comme distincts; et qui s'échangeraient entre eux quand on tournerait autour du centre du cercle. Nous pourrons nous placer tantôt à l'un, tantôt à l'autre de ces différents points de vue.

Nous pouvons envisager maintenant le domaine d'un ensemble de fonctions 
analytiques. Soient $F_{1}, F_{2}, \ldots, F_{p}$, un pareil ensemble. Un élément se composera d'un cercle de convergence $C$, et de $p$ séries de puissances $R_{1}, R_{2}, \ldots, R_{p}$, convergentes à l'intérieur de ce cercle, et représentant respectivement une des déterminations des $p$ fonctions $F_{1}, F_{2}, \ldots, F_{p}$. Deux éléments $C, R_{1}, R_{2}, \ldots, R_{p}$ et $C^{\prime}, R_{1}^{\prime}$, $\ldots, R_{p}^{\prime}$ sont contigus quand $C$ et $C^{\prime}$ ont une partie commune et quand on a dans cette partie commune $R_{1}=R_{1}^{\prime}, R_{2}=R_{2}^{\prime}, \ldots, R_{p}=R_{p}^{\prime}$. Rien à changer d'ailleurs à la définition du domaine principal.

Quelle relation y a-t-il entre le domaine principal $D$ de l'ensemble et les domaines principaux $D_{1}, D_{2}, \ldots, D_{p}$ des $p$ fonctions considérées individuellement? Considérons un élément de $D_{1}$ et un point de cet élément, il pourra se faire qu'en ce point une des fonctions $F_{2}, F_{3}, \ldots, F_{p}$ n'existe pas; dans ce cas ce point n'appartiendra pas à $D$; il pourra se faire aussi que l'une de ces $p-1$ fonctions admette plusieurs valeurs, $n$ par exemple; alors à ce point de $D_{1}$ correspondront $n$ points de $D$ qui devront être regardés comme distincts. Au contraire à tout point de $D$ correspondra toujours un point de $D_{1}$ et un seul, Nous pouvons alors construire un domaine intermédiaire, $D_{1}^{\prime}$, déduit de $D_{1}$ en supprimant tous les points de ce domaine auxquels ne correspondent aucun point de $D$; de sorte que $D_{1}^{\prime}$ est contenu dans $D_{1}$ et que $D$ est multiple de $D_{1}^{\prime}$.

Deux points d'un domaine quelconque seront infiniment voisins s'ils ont des coordonnées infiniment peu différentes, et s'ils appartiennent à un même élément de ce domaine. Cela suffit pour qu'on comprenne ce qu'on doit entendre par une ligne continue tracée dans un domaine, par une ligne fermée, par une aire continue dont tous les points font partie d'un domaine, et par la frontière de cette aire. Le domaine sera alors simplement connexe si toute courbe fermée tracée sur ce domaine est la frontière complète d'une aire continue dont tous les points font partie du domaine.

\section{§ 3. Formation de la fonction de Green.}

Considérons un domaine $D$ quelconque; soit $C_{0}$ un cercle représentant l'un des éléments de ce domaine, soit $O$ un point intérieur à $C_{0}$; nous allons définir d'abord la fonction initiale $u_{0}$.

Io. A l'intérieur de l'élément $C_{0}$.

Soit $O^{\prime}$ un point extérieur au cercle $C_{0}$, de telle façon que la droite $O O^{\prime}$ passe par le centre de ce cercle, et que le produit des distances de ce centre aux deux points $O$ et $O^{\prime}$ soit égal au carré du rayon du cercle. Alors si $P$ est

un point quelconque de la circonférence de $C_{0}$, le rapport $\frac{P O}{P O^{\prime}}$ sera égal à une 
constante $K$. C'est ce que nous exprimerons en disant que les points $O$ et $O^{\prime}$ sont inverses l'un de l'autre par rapport au cercle $C_{0}$.

Si alors $M$ est un point du domaine, intérieur à $C_{0}$, nous prendons pour la valeur de la fonction $u_{0}$ au point $M$ :

$$
u_{0}=\log \frac{M O^{\prime} . K}{M O}
$$

On voit que $u_{0}$ devient logarithmiquement infini au point $O$, est toujours, positif à l'intérieur de $C_{0}$ et nul sur la circonférence ce de cercle; $u_{0}$ peut être regardé comme le potentiel logarithmique dû à une masse + I placée au point $O$ et à des masses negatives réparties sur la circonférence de $\boldsymbol{C}_{\boldsymbol{0}}$.

$2^{\circ}$. Dans les éléments contigus à $C_{0}$.

Si $C_{1}$ est un élément contigu à $C_{0}$, on aura dans la partie commune à $C_{1}$ et à $C_{0}$

$$
u_{0}=\log \frac{M O^{\prime} . K}{M O}
$$

et en dehors de cette partie commune

$$
u_{0}=0
$$

$3^{\circ}$. Dans les éléments non-contigus à $C_{0}$, ou aura partout $u_{0}=0$.

On voit d'après cela que la fonction $u_{0}$ est partout positive ou nulle, qu'elle est continue, mais que ses dérivées ne le sont pas.

Nous allons maintenant définir une suite indéfinie de fonctions:

$$
u_{0}, u_{1}, u_{2}, \ldots, u_{n}, \ldots
$$

Ces fonctions, comme nous allons le voir, seront toutes positives ou nulles: elles seront finies, sauf au point $O$, où elles deviendront logarithmiquement infinies de telle façon que la différence $u_{n}-u_{0}$ reste finie. De plus elles seront continues mais leurs dérivées ne le seront pas. Et ceci nous amène à définir ce que nous appelons les masses génératrices. Ces masses sont réparties le long des lignes de discontinuité de ces dérivées. Soit dans un élément du domaine $\boldsymbol{L}$ une de ces lignes de discontinuité; la fonction $u$, d'après ce qui précède est continue, mais il n'en est pas de même de ses dérivées, et en particulier la «dérivée normale» $\frac{d u}{d n}$ relative à la ligne $L$ subit un saut brusque, quand on franchit cette ligne. C'est ce saut brusque divisé par $2 \pi$ qui représente la densité de la masse génératrice le long de la ligne $L$. Les masses ainsi réparties le long des lignes de discontinuité sont comme nous le verrons toutes négatives, et il faut $\mathrm{y}$ adjoindre une masse positive égale à $+\mathrm{I}$ et située au point $O$. 
Nous allons voir comment nous passons de l'une des fonctions de la série (1) à la suivante; comme nous connaissons $u_{0}$, toutes les autres fonctions de la série (I) se trouveront ainsi définies. Le passage de la fonction $u_{n}$ à la fonction $u_{n+1}$ se fera par la méthode du balayage, que j'ai exposée dans l'American Journal of Mathematics tome 12.

Considérons la fonction $u_{n}$, soit $C_{K}$ l'élément du domaine $D$ que nous nous proposons de balayer. Envisageons les masses génératrices négatives relatives à la fonction $u_{n}$ et situées à l'intérieur de $C_{K}$; elles sont réparties le long de certaines lignes de discontinuité $L$. Soit $d s$ un élément de l'une de ces lignes, et - $\mu d s$ la masse génératrice négative située sur cet élément. Alors nous aurons pour la valeur de la fonction $u_{n+1}-u_{n}$ au point $M$ :

I․ A l'intérieur de $C_{K}$ :

$$
u_{n+1}-u_{n}=\int \mu d s \log \frac{M Q^{\prime} \cdot P Q}{M Q \cdot P Q^{\prime}}
$$

$Q$ est le centre de gravité de l'élément $d s, Q^{\prime}$ est inverse de $Q$ par rapport au cercle $C_{K}$, le point $P$ est un point quelconque de la circonférence de $C_{K}$; quel que soit d'ailleurs ce point, le rapport $\frac{P Q}{P Q^{\prime}}$ conservera la même valeur, puisque $Q$ et $Q^{\prime}$ sont inverses par rapport à $C_{K}$. L'intégrale doit être étendue à tous les éléments de toutes les lignes $d s$ de discontinuité $L$ intérieures à $C_{K}$.

$2^{\circ}$. Dans un élément $C_{h}$ contigu à $C_{k}$.

On aura encore:

$$
u_{n+1}-u_{n}=\int \mu d s \log \frac{M Q^{\prime} \cdot P Q}{M Q \cdot P Q^{\prime}}
$$

dans la partie commune à $C_{h}$ et à $C_{k}$, et

$$
u_{n+1}-u_{n}=0
$$

en dehors de cette partie commune.

$3^{\circ}$. Dans un élément non contigu à $C_{k}$ on aura partont $u_{n+1}-u_{n}=0$.

Il résulte de là, que la fonction $u_{n+1}-u_{n}$ est partout positive ou nulle; qu'elle est continue; que c'est un potentiel logarithmique, engendré par des masses génératrices positives $\mu d s$ placées sur les divers éléments $d s$ des lignes de discontinuité $L$ intérieures à $C_{k}$, et par des masses négatives réparties sur la circonférence de $C_{k}$. La densité de cette matière génératrice négative en un point $P$ de cette circonférence est.

$$
\int \frac{\mu d s}{2 \pi}\left(\frac{\cos \varphi^{\prime}}{P Q^{\prime}}-\frac{\cos \varphi}{P Q}\right)
$$


$\%$ et $\varphi^{\prime}$ étant les angles que fait le rayon mené de $P$ au centre de $C_{k}$ avec les droites $P Q$ et $P Q^{\prime}$. On voit que cette densité est toujours finie quelle que soit la position du point $P$ sur la circonférence $C_{k}$.

Nous voyons alors que pour la fonction $u_{n+1}$, les masses négatives qui se trouvaient sur les lignes $L$ intérieures à $C_{k}$ ont disparu; car les masses négatives qui engendraient $u_{n}$ sont égales et contraires aux masses positives qui engendrent $u_{n+1}-u_{n}$. Les masses négatives qui se trouvaient à l'intérieur de $C_{k}$ en ont été balayées. Si le point $O$ est intérieur à $C_{k}$, il s'y trouvait une masse positive $+\mathrm{I}$, qui était l'une des masses génératrices de $u_{n}$. Le balayage ne porte pas sur cette masse positive qui subsiste et demeure l'une des masses génératrices de $u_{n+1}$. Ainsi aucune des masses génératrices de $u_{n+1}$ n'est à l'intérieur de $C_{k}$, sauf la masse positive + I qui pourrait se trouver au point $O$. Donc en aucun point de $C_{k}$, (sauf au point $O$, si ce point est intérieur à $C_{k}$ ) il n'y a de masse génératice de $u_{n+1}$. Donc en tout point de $C_{k}$ sauf en $O$, la fonction $u_{n+1}$ est une fonction harmonique, finie et continue ainsi que ses dérivées et satisfaisant à l'équation de LAPLACE $\Delta u_{n+1}=0$.

On voit de plus que les lignes de discontinuité $L$ ne sont autre chose que les circonférences des différents cercles qui représentent les éléments du domaine.

J'ai expliqué dans le mémoire cité, (American Journal, page 225) comment on peut choisir l'ordre des balayages de façon que chaque élément du domaine soit balayé une infinité de fois.

Nous avons donc une série de fonctions

$$
u_{1}, u_{2}, \ldots, u_{n}, \ldots
$$

toujours positives et toujours croissantes; c'est à dire que nous avons une série

$$
\sum\left(u_{n+1}-u_{n}\right)
$$

dont tous les termes sont positifs ou nuls. Deux hypothèses sont possibles; la série converge ou elle diverge. Voici ce que nous apprend à ce sujet le théorème de Harnack.

D'après ce théorème, si on a une série

$$
f_{1}+f_{2}+\cdots+f_{n}+\cdots
$$

et qu'à l'intérieur d'un certain cercle, le terme général $f_{n}$ soit une fonction harmonique et positive; si la série converge en un point intérieur au cercle, elle convergera en tout autre point intérieur au cercle; de plus la convergence sera uniforme et la série obtenue en la différentiant une ou plusieurs fois terme à terme sera aussi uniformément convergente.

Cela posé, considérons un élément $C_{k}$. Cet élément est balayé une infinité de fois. Soient 


$$
u_{\alpha_{1}}, u_{\alpha_{2}}, \ldots, u_{\alpha_{n}}, \ldots
$$

les fonctions obtenues immédiatement après ces différents balayages. Si le point $O$ n'est pas intérieur à $C_{k}$, toutes ces fonctions sont harmoniques à l'intérieur de $C_{k}$; en tout cas, que $O$ soit ou ne soit pas intérieur à $C_{k}$, les différences de ces fonctions seront harmoniques à l'intérieur de $C_{k}$. Donc la série

$$
\sum\left(u_{u_{n+1}}-u_{a, n}\right)
$$

a tous ses termes harmoniques et positifs et nous pouvons lui appliquer le théorème de HaRNaCK, si donc elle converge en un point de $C_{k}$, elle convergera dans ce cercle tout entier. Si la série (2) converge, $u_{\alpha n}$ tend vers une limite finie quand $n$ croît indéfiniment, ce qui montre que $u_{n}$ ne croît pas indéfiniment avec $n$, e'est à dire que la série (1) converge. La condition nécessaire et suffisante pour que (2) converge, c'est que (I) converge. Si done (I) converge en un point de $C_{k}$, elle convergera dans tout $C_{k}$, et on en déduit aisément que si elle converge en un point du domaine $D$, elle convergera dans ce domaine tout entier.

De plus d'après le théorème de Harnack, si la convergence a lieu, elle sera uniforme, et on pourra différentier la série terme à terme, une ou plusieurs fois, la convergence restant uniforme; de sorte que la somme de la série sera une fonction harmonique.

Nous sommes donc en présence de deux hypothèses seulement.

$1^{\circ}$. Ou bien $u_{n}$ croît indéfiniment arec $n$ et cela pour tous les points du domaine $D$.

$2^{0}$. Ou bien, pour tous les points de $D, u_{n}$ tend vers une limite $u$, et cette limite que nous appellerons fonction de Green est une fonction harmonique, sauf au point $O$; et dans le voisinage de $O$, la différence $u-u_{0}$ est harmonique. Cette fonction de GREEN est partout positive ou nulle.

Voyons quelle est l'influence de l'ordre des balayages. Je dis que la fonction $u$ ne dépend pas de l'ordre des balayages, pourvu que chaque élément soit balayé une infinité de fois. Soit

$$
\begin{aligned}
& u_{0}, u_{1}, u_{2}, \ldots, u_{n}, \ldots \\
& u_{0}^{\prime}, u_{1}^{\prime}, u_{2}^{\prime}, \ldots, u_{n}^{\prime}, \ldots
\end{aligned}
$$

deux suites de fonctions obtenues par balayages successifs et ayant respectivement pour limites $u$ et $u^{\prime}$. Observons que la fonction initiale est la même pour les deux suites:

$$
u_{0}=u_{0}^{\prime} .
$$


On a donc $u^{\prime}>u_{0}$ puisque $u^{\prime}>u_{0}^{\prime}$; je dis que si $u^{\prime}>u_{n}$, on aura également $u^{\prime}>u_{n+1}$. Si on passe de $u_{n}$ à $u_{n+1}$ en balayant l'élément $C_{k}$, on a en dehors de $C_{k}, u_{n}=u_{n+1}$ et par conséquent $u^{\prime}>u_{n+1}$. A l'intérieur de $C_{k}$, on voit que $u^{\prime}$ est une fonction harmonique, et il en est de même de $u_{n+1}$ puisque $C_{k}$ vient d'être balayé. Il en sera donc de $u^{\prime}-u_{n+1}$, même si $O$ est à l'intérieur de $C_{k}$. Or sur la circonférence de $C_{k}$ on a $u_{n+1}=u_{n}$, et par conséquent $u^{\prime}>u_{n+1}$. On aura donc encore à l'interieur de $C_{k}$

$$
u^{\prime}>u_{n+1} \text {. }
$$

On en conclut par récurrence que

et à la limite

$$
u^{\prime}>u_{n}
$$

On trouverait de même

$$
u^{\prime} \geq u
$$

$$
u>u_{n}^{\prime} \quad u \geqq u^{\prime}
$$

Done

$$
u=u^{\prime}
$$

Si dans la formation de la suite des $u_{n}^{\prime}$, tous les éléments n'étaient pas balayés une infinité de fois, ce raisonnement ne serait plus applicable, parce que la fonction $u^{\prime}$ ne serait pas partout harmonique, des masses génératrices pouvant rester dans des éléments qui auraient fini par ne pas être balayés. Mais la fonction $u$ resterait harmonique et on aurait toujours

$$
u>u_{n}^{\prime}, \quad u \geqq u^{\prime} .
$$

Peut-on appliquer les mêmes procédés, quand le domaine comprend ce que nous avons appelé un élément algébrique (c'est à dire un cercle multiple, vide supra $\$ 2$ in fine). Oui, mais il faut expliquer comment on peut balayer un pareil élément. Soit $C_{k}$ le cercle en question, $A$ son centre, et supposons que ce cercle soit $n^{\text {ple }}$, c'est à dire que chaque point intérieur à $C_{k}$ représente $n$ points du domaine regardés comme distincts et qui s'échangent entre eux quand on tourne antour de $A$. Nous construirons un autre cercle $C^{\prime \prime}$ ayant pour centre un point quelconque $B$; de telle façon qu'à tout point $M$ du cercle $\mathrm{C}_{k}$ de coordonnées polaires $\rho$ et $\omega$, corresponde un point $M^{\prime}$ du cercle $C^{\prime}$ de coordonnées polaires, $\sqrt[n]{\rho}$ et $\frac{\omega}{n}$. La fonction $u_{p}$ avant le balayage est une fonction harmonique des coordonnées de $M$, sauf le long de certaines lignes de discontinuité $L$ où il y aura des masses génératrices négatives. Ce sera donc aussi une fonction harmonique des coor- 
données de $M^{\prime}$ sauf le long det lignes $L^{\prime}$ formées par les points correspondants de ceux de $L$. Le long de ces lignes $L$, il y a aussi des masses génératrices négatives, et en effet la fonction $u_{p}$ considérée comme fonction des coordonnées du point $M^{\prime}$, est continue le long de ces lignes, mais ses dérivées premières ne le sont pas. Nous pourrons balayer par le procédé ordinaire, celles de ces masses négatives qui sont à l'intérieur de $C^{\prime}$, (puisque $C^{\prime}$ est un cercle simple, et non plus un cercle multiple comme $C_{k}$ ); on définira ainsi la fonction $u_{p+1}$. On voit que, en dehors de $C_{k}$ ou aura $u_{p}=u_{p+1}$; de même sur la circonférence de $C^{\prime}$ et par conséquent sur celle de $C_{k}$. A l'intérieur de $C_{k}$, on aura $u_{p+1}>u_{p}$ et $u_{p+1}$ sera harmonique. Ainsi nous pouvons faire la représentation conforme du cercle multiple $C_{k}$ sur le cercle simple $C^{\prime}$, et appliquer la méthode du balayage au cercle $C^{\prime}$. De même si nous avions des éléments infinis, nous ferions la représentation conforme de la partie du plan extérieure au cercle $C_{l}$, sur l'intérieur d'un cercle $C^{\prime}$ et nous appliquerions la méthode du balayage à ce cercle $C^{\prime \prime}$.

Comparons maintenant les fonctions de Grees $u$ et $u^{\prime}$ relatives à deux domaines $D$ et $D^{\prime}: I^{0}$ quand $D$ est contenu dans $D^{\prime}$. Soient alors

$$
\begin{aligned}
& u_{0}, u_{1}, \ldots, u_{n}, \ldots \\
& u_{0}^{\prime}, u_{1}^{\prime}, \ldots, u_{n}^{\prime}, \ldots
\end{aligned}
$$

nos deux suites de fonctions, formées par balayages successifs et relatives la I $^{\text {ere }} \grave{\mathrm{a}} D$, la $2^{\text {de }}$ à $D^{\prime}$. Nous pourrons supposer:

$$
u_{0}=u_{0}^{\prime}
$$

et en effet comme $D$ est contenu dans $D^{\prime}$, nous pourrons supposer que le cercle $C_{0}$ qui est un élément de $D$, est aussi un élément de $D^{\prime}$. Nous aurons alors à l'intérieur de $C_{0}$

et en dehors de $C_{0}$

$$
u_{0}=u_{0}^{\prime}>0
$$

$$
u_{0}=u_{0}^{\prime}=0 \text {. }
$$

Si la ${ }^{\text {ere }}$ suite est convergente, nous appellerons $u$ sa limite et de même si la $2^{\text {de }}$ suite converge, sa limite s'appellera $u^{\prime}$. La fonction $u^{\prime}$ est harmonique dans $D^{\prime}$ et par conséquent dans $D$, (sauf au point $O$ où c'est $u^{\prime}-u_{0}$ qui est harmonique); elle est d'ailleurs positive; on démontrerait comme plus haut que l'on a $u^{\prime}>u_{0}$, que l'on a $u^{\prime}>u_{n+1}$ si $u^{\prime}>u_{n}$, que par conséquent quel que soit $n$ on a $u^{\prime}>u_{n}$ et à la limite:

$$
u^{\prime} \geqq u
$$

D'où cette conséquence, si la suite des $u_{n}^{\prime}$ converge pour le domaine $D^{\prime}$, la suite des $u_{n}$ relative ì un domaine $D$ contenu dans $D^{\prime}$ convergera a fortiori. 
$2^{\circ}$. Quand le domaine $D$ est multiple de $D^{\prime}$.

Cette fois à tout point de $D^{\prime}$ correspondent plusieurs points de $D$; si nous reprenons nos deux séries, il faut supposer qu'aux différents points de $D$ qui correspondent à un même point de $D^{\prime}$, la fonction $u_{n}^{\prime}$ reprend la même valeur, mais que la fonction $u_{n}$ ne reprend pas en général la même valeur. Soit $C_{0}$ celui des éléments de $D$ qui contient le point $O$; il y aura alors d'autres points $O_{1}, O_{2}, \ldots, O_{p}, \ldots q$ qui correspondront au même point de $D^{\prime}$ que le point o; et on pourra les regarder comme appartenant à autant de cercles $C_{0}^{1}, C_{0}^{2}, \ldots, C_{0}^{p}, \ldots$ qui seront des éléments distincts de $D$, mais qui correspondront à un même élément de $D^{\prime}$. Alors la fonction $u_{n}^{\prime}$ deviendra logarithmiquement infinie, non seulement en $O$, mais en $O_{1}, O_{2}, \ldots$, tandis que la fonction $u_{n}$ ne deviendra infinie qu'en $O$.

Alors nous aurons dans le cercle $C_{0}$

$$
u_{0}=u_{0}>0
$$

dans les autres cercles $C_{0}^{1}, C_{0}^{2}$, etc.

et en dehors de ces cercles

$$
u_{0}^{\prime}>0, \quad u_{0}=0
$$

$$
u_{0}=u_{0}^{\prime}=0
$$

Si donc la suite des $u_{n}^{\prime}$ converge on aura:

$$
u^{\prime}>u_{0}^{\prime} \geqq u_{0}
$$

et en raisonnant comme plus haut on trouvera:

$$
u^{\prime}>u_{n}
$$

Une observation toutefois; nous nous sommes appuyés plus haut sur ce que la différence $u^{\prime}-u_{n+1}$ est harmonique à l'intérieur du cercle $C_{k}$ que l'on vient de balayer; sachant de plus que cette différence est positive sur la circonférence de ce cercle, nous en avons conclu qu'elle reste positive à l'interieur de ce cercle. Ici il peut se faire que $u^{\prime}-u_{n+1}$ devienne infinie, puisque $u^{\prime}$ devient infinie aux points $O_{p}$ différents de $O$, tandis que $u_{n+1}$ reste finie, mais alors cette différence tend vers $+\infty$ et non pas vers $-\infty$ de sorte que la conclusion subsiste.

On aura donc à la limite

$$
u^{\prime} \geq u
$$

Si donc la serie converge pour un domaine $D^{\prime}$ elle convergera a fortiori pour un domaine $D$ multiple de $D^{\prime}$.

$3^{\circ}$ quand enfin à tout point de $D$ correspond un point de $D^{\prime}$ et un seul, tandis qu'à un point de $D^{\prime}$ peuvent correspondre soit zéro, soit un, soit plusieurs points de $D$. 
Dans ce cas il existe un domaine intermédiaire $D^{\prime \prime}$ tel que $D$ soit multiple de $D^{\prime \prime}$ et $D^{\prime \prime}$ contenu dans $D^{\prime}$.

$\mathrm{Si}$ alors la serie converge pour $D^{\prime}$, elle convergera pour $D^{\prime \prime}$, et par conséquent pour $D$.

On voit que la démonstration du mémoire cité (Bull. Soc. Math.) a été simplifiée par l'application de la méthode du balayage et surtout par celle du théorème de HaRnaCK, qui permet de supprimer toutes les discussions relatives à l'uniformité de la convergence.

\section{$\$ 4$. Introduction de la fonction auxiliaire majorante.}

Considérons un système de fonctions

$$
y_{1}, y_{2}, \ldots, y_{p}
$$

et soit $D$ le domaine principal de ce système; soit $D_{1}$ le domaine principal de l'une des fonctions $y_{1}$; d'après ce que nous avons vu plus haut, à chaque point de $D$ correspond toujours un point de $D_{1}$ et un seul; mais à un point de $D_{1}$ peuvent correspondre zéro, un ou plusieurs points de $D$. Il existe donc un domaine intermediaire $D_{1}^{\prime}$, contenu dans $D_{1}$ et dont $D$ est multiple, et il en résulte comme nous venons de le voir, que si la série converge pour $D_{1}$, elle convergera également pour $D$, et la fonction de GreEs existera pour $D$.

Soit maintenant $D^{\prime}$ un domaine secondaire de notre système de fonctions, il sera multiple de $D$ de sorte que la fonction de GreEn existera également pour $D^{\prime}$.

Cela posé il y a des fonctions $y_{1}$ pour le domaine principal desquelles la fonction de Grees existe certainement. Considérons en effet une fonction fuchsienne quelconque

$$
x=f(z)
$$

n'existant qu'à l'intérieur du cercle fondamental, de centre $O$ et de rayon I. Résolvons l'équation $x=f(z)$ par rapport à $z$ et soit

$$
z=f(x)
$$

$\varphi(x)$ sera une fonction multiforme dont on peut former le domaine principal $D_{1}$ Considérons sur ce domaine $D_{1}$ la fonction

$$
t=\log \frac{1}{|z|}
$$

ce sera la fonction de GREEN cherchée; elle est en effet toujours positive, et toujours harmonique, sauf en un seul point du domaine $D_{1}$, celui pour lequel on a $z=0$, et en ce point elle devient logarithmiquement infinie. 
Nos fonctions $u_{n}$ du $\S$ précédent sont toujours plus petites que $t$, et cela est vrai des fonctions $u_{n}$ relatives soit au domaine $D_{1}$ lui-même, soit à tout domaine contenu dans $D_{1}$, on multiples d'un domaine contenu dans $D_{1}$. Si done notre système (I) comprend la fonction

$$
y_{1}=z=\varphi(x)
$$

la suite des $u_{n}$ converge et la fonction de GrEen existe, soit pour le domaine principal $D$, soit pour un domaine secondaire $D^{\prime}$ quelconque de ce système (I). La fonction $t$ joue le même rôle que celle que nous avons désignée par la mêm lettre dans le mémoire cité (Bull. Soc. Math.). Elle permet de montrer par l'inégalité

$$
u_{n}<t
$$

que la série converge sauf pour les points où $t$ est infini. Ces points sont les points de $D$ qui correspondent au point $z=0$ de $D_{1}$; en général, il y en a plusieurs, l'un est le point $O$, les autres pourront s'appeler $O_{1}, O_{2}, \ldots$

La fonction de GrEen, d'après sa définition, doit devenir infinie au point $O$, mais rester finie en $O_{1}, O_{2}$ etc.; seulement le théorème de Harnack nous apprend que si la série converge en un point du domaine $D$, elle convergera dans tous les autres points de ce même domaine, sauf au point $O$. Or elle converge en tous les points où $t$ est fini; done elle convergera également en $O_{1}, O_{2}, \ldots$ Nous sommes ainsi dispensés de la considération des fonctions $t_{i}$ qui dans le mémoire cité, avaient été précisément introduites pour démontrer la convergence aux points tels que $O_{1}, O_{2}, \ldots$

Supposons maintenant que dans notre système (I) ne figure pas de fonction de cette forme, c'est à dire qui soient l'inverse d'une fonction fuchsienne. Nous formerons alors un système! ( $\mathrm{I}$ bis), obtenu en adjoignant au système (I) une $p+\mathrm{I}^{\mathrm{e}}$ fonction

$$
y_{p+1}=z=\varphi(x)
$$

qui soit l'inverse d'une fonction fuchsienne.

Soit $\Delta$ le domaine principal du système (I bis), et $\Delta^{\prime}$ un domaine secondaire de ce même système. Alors $\Delta^{\prime}$ et $\Delta$ sont multiples d'un domaine $\delta$ contenu dans le domaine principal $D$ du système (I).

Nous sommes certains alors que la fonction de GREEN existe pour $\Delta$ et $\Delta^{\prime}$ puisque le système ( $\mathrm{I}$ bis) contient une fonction inverse d'une fonction fuchsienne; nous verrons que l'existence de cette fonction de GrEen, permet d'uniformiser les fonctions

$$
y_{1}, y_{2}, \ldots, y_{p}, y_{p+1}
$$

Acta mathematica. 31. Imprimé le 19 mars 1907. 
c'est à dire d'exprimer ces $p+1$ quantités en fonctions uniformes d'une même variable auxiliaire $\zeta$; mais une difficulté peut se présenter; le champ dans lequel cette uniformisation sera réalisée, comprendra tous les points pour lesquels les $p+1$ fonctions existent, c'est à dire tous les points qui ont un correspondant dans $\Delta$, c'est à dire tous les points de $\delta$. Elle ne le sera pas pour tous les points pour lesquelles les $p$ premières fonctions données existent (abstraction faite de la $p+\mathrm{I}^{\mathrm{\theta}}$ fonction arbitrairement adjointe) c'est à dire pour tous les points de $D$, si $\delta$ est plus petit que $D$. La solution pourrait donc paraître imparfaite et c'est ce qui me conduit à la discussion suivante.

On peut faire plusieurs hypothèses au sujet de la fonction fuchsienne dont l'inverse est $y_{p+1}$.

$I^{0}$. Nous pouvons supposer que le polygone générateur de cette fonction fuchsienne a des sommets sur le cercle fondamental, c'est à dire qu'il est de la $2^{\mathrm{e}}$ ou de la $6^{\theta}$ familles. C'est ce qui arrive par exemple si l'on choisit pour cette onction fuchsienne la fonction modulaire comme je l'ai fait dans le mémoire cité. On sait que cette fonction ne peut prendre aucune des valeurs $0,1, \infty$ de sorte que la fonction inverse

$$
y_{p+1}=\varphi(x)
$$

n'existe pas pour $x=0, x=\mathrm{I}, x=\infty$; si donc il y a dans le domaine $D$ des points pour lesquels $x$ prend l'une de ces 3 valeurs, ces points doivent être exclus du domaine $\delta$; ce sont des Ausnahmsstellen.

$2^{0}$. Nous pouvons supposer que le polygone générateur est tout entier à l'intérieur du cercle fondamental, c'est à dire que la fonction fuchsienne est de la I ere famille. Dans ce cas, cette fonction peut prendre toutes les valeurs possibles; la fonction inverse

$$
y_{p+1}=\varphi(x)
$$

existe pour toutes les valeurs de $x$. Il arrivera toutefois que cette fonction présentera des points de ramification algébrique. Si nous nous placions au point de vue que nous avions d'abord adopté, c'est à dire si nous ne voulions composer nos domaines que d'éléments holomorphes ou polaires, ces points de ramification seraient en dehors du domaine principal de cette fonction $y_{p+1}$; il en sera plus de même si au contraire nous admettons les éléments algébriques; à tout point de $D$ correspondra un point du domaine de $y_{p+1}$ et par conséquent un point de $\Delta$. Les domaines $\delta$ et $D$ seront identiques; $\Delta$ et $\Delta^{\prime}$ seront multiples de $D$ et il n'y aura plus d'Ausnahmsstellen.

Mais il y a quelque chose de plus; soient $\Delta$ et $D$ deux domaines quelconques et supposons que $\Delta$ soit multiple de $D$. Nous dirons que $\Delta$ est régulièrement 
multiple de $D$, s'il satisfait à la condition suivante. Soit $M$ un point de $D$; soient

$$
M_{1}, M_{2}, \ldots
$$

les points correspondants de $\Delta$. Soit $M^{\prime}$ un autre point de $D$ infiniment voisin de $M$; nous supposerons que parmi les points de $\Delta$ qui correspondent à $M^{\prime}$, il $y$ en ait un qui soit infiniment voisin de $M_{1}$, un qui soit infiniment voisin de $M_{2}, \ldots$

$\mathrm{Si}$ cette condition est remplie, c'est à dire si $\Delta$ est régulièrement multiple de $D$, il est clair que, si à un certain point $M$ de $D$ correspondent un nombre fini $n$ de points de $\Delta$, alors à tout autre point de $D$ correspondront le même nombre fini $n$ de points de $\Delta$.

Revenons maintenant aux domaines principaux $\Delta$ et $D$ des systèmes (I bis) et (I). Je dis que $\Delta$ est régulièrement multiple de $D$ (si l'on admet des éléments algébriques, et si la fonction fuchsienne auxiliaire est de la $I^{\text {ere }}$ famille.) Soit en effet $M$ un point du domaine $D$, et $x$ la valeur correspondante de cette variable. Pour cette valeur $x$, la fonction $y_{p+1}$ existe et peut prendre une infinité de valeurs

$$
\alpha_{1}, \alpha_{2}, \ldots, \alpha_{q}, \ldots
$$

qui se déduisent de l'une d'entre elles, $\alpha_{1}$ par exemple, en appliquant à cette quantité $\alpha_{1}$ les diverses substitutions linéaires du groupe fuchsien qui engendre la fonction fuchsienne auxiliaire.

Au point $M$ de $D$, correspondront une infinité de points de $\Delta$, qui j'appellerai

$$
\left(M, \alpha_{1}\right),\left(M, \alpha_{2}\right), \ldots,\left(M, \alpha_{q}\right), \ldots
$$

et qui correspondront aux différentes, valeurs de la fonction $y_{p+1}$.

Soit maintenant $M^{\prime}$ un autre point de $D$, infiniment voisin de $M$; soit $x+\varepsilon$ la valeur correspondante de $x$, et

$$
\beta_{1}, \beta_{2}, \ldots, \beta_{q}, \ldots
$$

- les déterminations correspondantes de $y_{p+1}$. Au point $M^{\prime}$ de $D$ correspondront une infinité de points de $\Delta$ qui sont:

$$
\left(M^{\prime}, \beta_{1}\right),\left(M^{\prime}, \beta_{2}\right), \ldots,\left(M^{\prime}, \beta_{q}\right), \ldots
$$

Ce qu'il s'agit de démontrer, e'est que parmi ces points il y en a un qui diffère infiniment peu de $\left(M, \alpha_{q}\right)$, et comme $M^{\prime}$ diffère très peu de $M$ par hypothèse, il suffit d'établir que parmi les déterminations de $\varphi(x+\varepsilon)$, il y en a une, $\beta_{q}$ par exemple, qui diffère infiniment peu de la détermination $\alpha_{q}$ de $\varphi(x)$. Or cela paraîtra évident pour peu qu'on réfléchisse aux propriétés des fonctions fuchsiennes de la I ere famille. 
Soit maintenant $D$ un domaine quelconque; je dis que nous pourrons trouver un domaine $\Delta$, régulièrement multiple de $D$ et simplement connexe. Soit en effet $C_{0}$ l'élément initial de $D, C$ un élément quelconque, $M$ un point intérieur à $C$, $M_{0}$ un point intérieur à $C_{0}$. On peut aller sur le domaine $D$ de $M_{0}$ en $M$ par plusieurs chemins; envisageons deux de ces chemins; ils pourront être équivalents, c'est à dire qu'ils pourront limiter une aire continue située sur $D$; mais ils pourront aussi ne pas l'être, à moins que $D$ ne soit simplement connexe.

Cela posé, définissons le domaine $\Delta$; un point de ce domaine sera caractérisé par le point $M$ de $D$ qui lui correspond, et par le chemin par lequel on est venu de $M_{0}$ en $M$; pour que deux points de $\Delta$ ainsi caractérisés soient identiques, il faudra et il suffira qu'ils correspondent à un même point $M$ de $D$ et qu'on soit venu de $M_{0}$ en $M$ par deux chemins équivalents. Il est clair que $\Delta$ est simplement connexe, je dis qu'il est régulièrement multiple de $D$. Soit en effet $M$ un point de $D$, appartenant à un élément $C, M_{0} N M, M_{0} N^{\prime} M$, deux chemins allant de $M_{0} \grave{a} M$ et que je supposerai équivalents. Ainsi se trouvera défini un point de $\Delta$ que j'appellerai $\left(M, M_{0} N M\right)$. Ce qu'il s'agit de démontrer, e'est que si $\mathbf{M}^{\prime}$ est un point de $D$, très voisin de $M$, il y aura sur $\Delta$ un point très voisin de $\left(M, M_{0} N M\right)$ et qui correspondra à $M^{\prime}$. Et en effet, $M^{\prime}$ étant très voisin de $M$, pourra être joint à $M$ par un arc $M M^{\prime}$ très petit; en l'adjoignant au chemin $M_{0} N M$, on obtiendra un chemin $M_{0} N M M^{\prime}$ qui en diffèrera très peu; et alors le point $\left(M^{\prime}, M_{0} N M M^{\prime}\right)$ est un point de $\Delta$ qui correspond à $M^{\prime}$ et qui est très voisin de $\left(M, M_{0} N M\right), \ldots$ C. Q. F. D. Ce qui justifie ce résultat, c'est que si deux chemins $M_{0} N M$ et $M_{0} N^{\prime} M$ sont équivalents, il en est de même des deux chemins $M_{0} N M M^{\prime}$ et $M_{0} N^{\prime} M M^{\prime}$.

En résumé, étant donné un système (I) quelconque de fonctions multiformes, et $D$ le domaine principal de ce système, on peut toujours former un domaine régulièrement multiple de $D$, simplement connexe, et pour lequel la fonction de Green existe.

Et en effet, formons une fonction $y_{p+1}=p(x)$, inverse d'une fonction

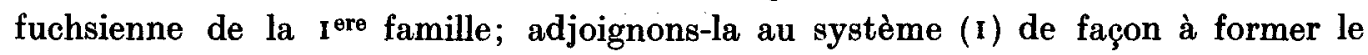
système ( $\mathrm{l}$ bis); le domaine principal $\Delta$ de ce système sera régulièrement multiple de $D$, ̀̀ la condition que nous admettions des éléments algébriques. De plus $\Delta$ sera multiple d'un domaine contenu dans le domaine principal de $y_{p+1}$ et comme la fonction de GreEN existe pour ce dernier domaine, elle existera a fortiori pour $\Delta$. Nous avons vu en effet que la présence d'éléments algébriques n'empêche pas l'application de la méthode du balayage.

Nous pouvons ensuite construire un domaine $\Delta^{\prime}$, régulièrement multiple de $\Delta$ et simplement connexe. Etant régulièrement multiple de $\Delta$, il le sera de $D$. De plus la fonction de Green existant pour $\Delta$, existera a fortiori pour $\Delta^{\prime}$. 


\section{5. Les fonctions $\boldsymbol{v}$ et $\boldsymbol{z}$.}

Soit un domaine $D$ quelconque, admettant une fonction de Green.

Nous allons maintenant comme dans le mémoire cité, définir les fonctions $v$ et $v_{n}$. Nous avons défini déjà les fonctions $u$ et $u_{n}$; si $x=\xi+i \eta$ est la variable complexe envisagée, nous aurons:

Les équations

$$
\begin{gathered}
\Delta u=\frac{d^{2} u}{d \xi^{2}}+\frac{d^{2} u}{d \eta^{2}}=0 \\
\Delta u_{n}=\frac{d^{2} u_{n}}{d \xi^{2}}+\frac{d^{2} u_{n}}{d \eta^{2}}=0 .
\end{gathered}
$$

$$
\left\{\begin{array}{l}
\frac{d v}{d \xi}=-\frac{d u}{d \eta}, \frac{d u}{d \xi}=\frac{d v}{d \eta} \\
\frac{d v_{n}}{d \xi}=-\frac{d u_{n}}{d \eta}, \frac{d u_{n}}{d \xi}=\frac{d v_{n}}{d \eta}
\end{array}\right.
$$

sont donc compatibles et peuvent servir à définir $v$ et $v_{n}$. On achève de définir ces fonctions en leur imposant la condition de s'annuler en un point $A$ différent de $O$; et alors nous aurons

$$
v=\int_{A}\left(\frac{d u}{d \xi} d \eta-\frac{d u}{d \eta} d \xi\right), \quad v_{n}=\int_{A}\left(\frac{d u_{n}}{d \xi} d \eta-\frac{d u_{n}}{d \eta} d \xi\right) .
$$

On voit tout de suite que les suites $\frac{d u_{n}}{d \xi}, \frac{d u_{n}}{d \eta}$ convergeant uniformément (en vertu du théorème de HARNACK) et ayant pour limites $\frac{d u}{d \xi}, \frac{d u}{d \eta}$, la suite $v_{n}$ convergera uniformément et aura pour limite $v$; nous reviendrons plus loin sur ce résultat pour le préciser. Posons ensuite, toujours comme dans le mémoire cité:

$$
z=e^{-(u+i v)}, \quad z_{n}=e^{-\left(u_{n}+i v_{n}\right)}
$$

il est clair que la suite $z_{n}$ a pour limite $z$. Le point essentiel, c'est que la fonction $z$ est uniforme sur le domaine $D$ quand ce domaine est simplement connexe.

En effet revenons aux intégrales (2) qui définissent $v$, ces intégrales peuvent admettre deux sortes de périodes: $1^{\circ}$ une période polaire, relative au point $O$ qui est un infini pour les fonctions sous le signe $f$. Cette période est égale à $2 \pi$. et $2^{\circ}$, si $D$ n'est pas simplement connexe, des périodes cycliques relatives aux chemins d'intégration qui seraient fermés sans être équivalents à zéro. Si $D$ est 
simplement connexe, ces dernières périodes n'existent pas, de sorte que $v$ n'admet que la période $2 \pi$; mais quand $v$ augmente de $2 \pi, z$ ne change pas. Cette fonction est donc_uniforme.

C. Q. F. D.

Mais il $\mathrm{y}$ a lieu d'examiner de plus près la formation de ces fonctions $v_{n}$ et $z_{n}$. Soit d'abord $C_{0}$ l'élément initial, celui qui contient le point $O$. Nous aurons à l'extérieur de $C_{0}: z_{0}=\mathrm{I}$ et à l'intérieur de $C_{0}$

$$
z_{0}=\frac{x-a}{x-a^{\prime}} \frac{X-a^{\prime}}{\bar{X}-a}
$$

expression dont je vais expliquer la signification: $x$ est la variable indépendante, c'est l'affixe du point $M$ de coordonnées courantes $\xi$ et $\eta$; $a$ est l'affixe du point $O, a^{\prime}$ celle du point $O^{\prime}$ inverse de $O$ par rapport au cercle $C_{0}$, de telle sorte que l'équation de la circonférence de $C_{0}$ soit:

$$
\left|\frac{x-a}{x-a^{\prime}}\right|=\text { const. }
$$

$X$ sera l'affixe d'un point quelconque de cette circonférence, il en résulte que la fonction $z_{0}$ n'est pas entièrement définie, puisque nous pouvons choisir arbitrairement $X$ sur cette circonférence; $z_{0}$ n'est définie qu'à un facteur constant près de module un.

Ayant ainsi défini $z_{0}$, voyons comment les balayages successifs vont nous permettre de passer de $z_{n}$ à $z_{n+1}$. Nous remarquerons que les fonctions $z_{n}$ peuvent présenter des discontinuités le long des lignes $L$, c'est à dire le long des circonférences des différents éléments; voyons quelle est la nature de ces discontinuités. D'abord $u_{n}$ est continu; il en résulte que quand on franchit une des lignes de discontinuité, le module de $z_{n}$ ne change pas, mais il n'en est pas de même de son argument $-v_{n}$. Les dérivées de $u_{n}$ sont au contraire discontinues. Soit $d s$ un élément de l'arc d'une des lignes $L, d \nu$ un élément normal à cette ligne; soient

$$
\frac{d u_{n}^{0}}{d s}, \frac{d u_{n}^{0}}{d \nu}, \frac{d u_{n}^{1}}{d s}, \frac{d u_{n}^{1}}{d \nu}
$$

les dérivées de $u_{n}$ prises le long de $L$, ou le long de la normale à $L$; l'exposant o correspondant à l'un des côtés de la ligne $L$, l'exposant I à l'autre côté. De même pour les dérivées de $v_{n}$. On aura:

$$
\frac{d u_{n}^{0}}{d s}=\frac{d u_{n}^{1}}{d s}, \frac{d u_{n}^{0}}{d \nu}=\frac{d u_{n}^{1}}{d \nu}+2 \pi m^{\prime}
$$


en désignant par $d m$ ce que nous avons appelé la masse génératrice située sur $d s$, et en posant $m^{\prime}=\frac{d m}{d s}$ de façon que $m^{\prime}$ représente la densité de cette matière génératrice. D'autre part les équations (I) nous donnent:

$$
\frac{d u_{n}}{d s}=-\frac{d v_{n}}{d \nu} ; \frac{d u_{n}}{d \nu}=\frac{d v_{n}}{d s}
$$

et on en conclut:

$$
\frac{d v_{n}^{0}}{d \nu}=\frac{d v_{n}^{1}}{d \nu}, \frac{d v_{n}^{0}}{d s}=\frac{d v_{n}^{1}}{d s}+2 \pi m^{\prime}
$$

Cela posé, supposons que pour passer de $z_{n}$ à $z_{n+1}$ on veuille balayer un certain élément $C_{k}$, et je supposerai d'abord qu'il s'agisse d'un élément holomorphe ou polaire. A l'extérieur de $C_{k}$ nous aurons:

et à l'intérieur de $C_{k}$ :

$$
z_{n+1}=z_{n}
$$

$$
\log \frac{z_{n+1}}{z_{n}}=\int \log \frac{(x-\alpha)\left(A-\alpha^{\prime}\right)}{\left(x-\alpha^{\prime}\right)(A-\alpha)} d m^{\prime}
$$

Dans cette expression, $x$ est la variable indépendante; $\alpha$ est l'affixe d'un point d'une ligne de discontinuité $L$ situé à l'intérieur de $C_{k} ; \alpha^{\prime}$ est l'affixe du point inverse de a par rapport à $C_{k}$, de telle façon que la circonférence de $C_{k}$ ait pour équation

$$
\left|\frac{x-\alpha}{x-\alpha^{\prime}}\right|=\text { const. }
$$

$A$ est l'affixe d'un point quelconque de la circonférence de $C_{k}$, choisi arbitrairement, mais une fois pour toutes; $d m^{\prime}$ est la masse génératrice située sur l'arc infiniment petit $d s$ de la ligne $L$, arc dont le centre de gravité est $\alpha$. Enfin l'intégration doit être étendue à toutes les lignes de discontinuité intérieures à $C_{k}$. A cause des équations (3) nous pouvons encore écrire:

$$
\log \frac{z_{n+1}}{z_{n}}=\int \log \frac{(x-\alpha)\left(A-\alpha^{\prime}\right)}{\left(x-\alpha^{\prime}\right)(A-\alpha)} d\left(\frac{v_{n}^{0}-\overline{v_{n}^{2}}}{2 \pi}\right)
$$

$v_{n}^{0}$ et $v_{n}^{1}$ étant les valeurs de la fonction $v_{n}$ de part et d'autre de la ligne de discontinuité et dans le voisinage du point $\alpha$ de cette ligne.

Si $C_{k}$ est un élément infini, c'est à dire comprenant la partie du plan extérieure à la circonférence de $C_{k}$, nous aurons en dehors de l'élément $C_{k}$; $y_{n+1}=z_{n}$, et dans l'élément $C_{k}$, qui est tout entier à l'extérieur de la circonférence de $C_{k}$ : 
$(4$ ter $)$

$$
\log \frac{z_{n+1}}{z_{n}}=\int \log \frac{(x-\alpha)\left(A-\alpha^{\prime}\right)}{\left(x-\alpha^{\prime}\right)(A-\alpha)} d\left(\frac{v_{n}^{1}-v_{n}^{0}}{2 \pi}\right)
$$

Si enfin $C_{k}$ est un élément algébrique et si $C_{k}$ est un cercle $p^{\text {ple }}$, ou aura à l'extérieur de $C_{k}: z_{n+1}=z_{n}$ et à l'intérieur de $C_{k}$ :

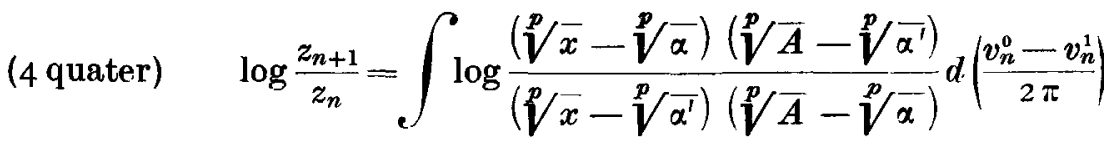

Ou voit qu'à cause des points $A$ qui peuvent être arbitrairement choisis sur la circonférence de l'élément $C_{k}$, les fonctions $z_{n}$ successives ne sont définies qu'à un facteur constant près de module I; et encore ce facteur n'est constant que si l'on ne franchit pas une des lignes de discontinuité, mais il peut varier brusquement quand on franchit ces lignes.

Supposons un chemin ne franchissant et ne touchant aucune de ces lignes de discontinuité, c'est à dire aucune des circonférences des différents éléments, et envisageons l'intégrale:

$$
\int d v_{n}=\int\left(\frac{d u_{n}}{d \xi} d \eta-\frac{d u_{n}}{d \eta} d \xi\right)
$$

prise le long d'un de ces chemins. Puisque le chemin n'a aucun point commun avec aucune des lignes de discontinuité, on peut construire une aire où il n'y aura aucune ligne de discontinuité et qui contiendra notre chemin. Dans cette aire toutes les fonctions $u_{n}$ sont harmoniques et on peut leur appliquer le théorème de HARNACK; donc la suite des $\frac{d u_{n}}{d \xi}$ et celle des $\frac{d u_{n}}{d \eta}$ convergent uniformément, de sorte que $\int d v_{n}$ tend vers $\int d v$.

$\mathrm{Si}$ nous supposons maintenant que notre chemin, tout entier contenu à l'intérieur d'un élément $C_{k}$, coupe une ligne de discontinuité $L$; le même raisonnement n'est plus immédiatement applicable. Et en effet les fonctions $u_{n}$ sont bien toutes positives, croissent avec $n$; de plus elles sont harmoniques dans l'aire envisagée, si l'on donne à $n$ certaines valeurs en nombre infini, à savoir celles qui correspondent aux balayages successifs de l'élément $C_{k}$, parce qu'après chacun de ces balayages, il n'y a plus de masses génératrices dans $C_{k}$. Donc par le théorème de HaRNaCK le suite des $u_{n}$ et celle des $\frac{d u_{n}}{d \xi}$ convergent uniformément pourvu qu'on ne donne à $n$ que ces valeurs particulières. Majs comme $u_{n}$ croît avec $n$, nous pouvons en conclure que la suite des $u_{n}$ convergera encore uniformément pour toutes les valeurs entières de $n$; seulement la même conclusion 
ne s'impose pas en ce qui concerne les dérivées $\frac{d u_{n}}{d \xi}$, qui ne vont pas nécessaireen croissant avec $n$. Décomposons notre chemin en deux parties séparées l'une de l'autre par le point d'intersection $P$ du chemin et de la ligne $L$. Il suffirait de démontrer le théorème pour ces deux parties. Soit $P M$ l'une de ces parties; si nous pouvions assigner aux dérivées de $u_{n}$ une limite supérieure $\lambda$, même au point $P$, le théorème serait démontré. En effet, nous voulons démontrer que l'on peut prendre $n$ assez grand pour que

quel que soit $\varepsilon$.

$$
\int_{P M}\left(d v-d v_{n}\right)<\varepsilon
$$

Pour cela nous diviserons $P M$ en deux parties $P N+N M$; si $s$ est la longueur de l'arc $P N$, et $\lambda$ la limite supérieure dont nous venons de parler, nous aurons

$$
\int_{P M}\left(d v-d v_{n}\right)<s \lambda
$$

et nous pourrons prendre $N$ assez près de $P$ pour que $s \lambda<\frac{\varepsilon}{2}$, et le point $N$ une fois choisi, comme $N M$ ne va plus jusqu'à la ligne $L$, nous pourrions prendre $n$ assez grand pour que

$$
\int_{N M}\left(d v-d v_{n}\right)<\frac{\varepsilon}{2}
$$

Nous sommes done conduits à chercher cette limite supérieure $\lambda$ et pour cela à étudier la façon dont les masses se distribuent sur les lignes $L$. Soit une masse $m$ située á l'intérieur d'un cercle $C_{k}$, en $A$; soit $B$ un point de la circonférence $C_{k}$, et $R$ le rayon de cette circonférence, et $O$ son centre. Balayons le cercle $C_{k}$, cette masse $m$ va se répartir sur toute la circonférence $C_{k}$; soit $\delta$ la densité de la matière ainsi répartie dans le voisinage de $B$. On voit que $\frac{\delta}{m}$ est une fonction des trois distances $O A, O B, A B$, et que cette fonction ne devient infinie que pour $A B=0$. Si le point $A$ décrit une ligne de discontinuité $L$ intérieure à $C_{k}$, et si $B$ est un point fixe de la circonférence $C_{k}$ non situé sur $L$, nous pourrons done assigner une limite supérieure $\grave{\mathbf{a}} \frac{\delta}{\mathrm{m}}$.

Supposons donc que $B$ soit un point d'une circonférence $C_{k}$, mais ne soit pas à l'intersection de $C_{k}$ avec une autre ligne de discontinuité; je me propose de chercher une limite supérieure de la densité de la matière attirante en ce 
point. Cette densité ne peut varier que dans deux cas: $1^{\circ}$ quand on balaye $C_{k}$. $2^{\circ}$ quand on balaye l'un des éléments dont fait partie $B$. Dans le $2^{\mathrm{d}}$ cas cette densité devient nulle. Dans le ${ }^{\text {er }}$ cas une masse négative au plus égale à I en valeur absolue est répartie en différents points $A$ de $C_{k}$; on la balaye vers la circonférence de $C_{k}$; les points $A$ sont sur les lignes de discontinuité intérieures à $C_{k}$, et comme le point $B$ n'est pas sur une de ces lignes, nous pouvons assigner une limite supérieure à la distance $A B$, et par conséquent d'après ce qui précède au rapport $\frac{\delta}{m}$, de l'accroissement $\delta$ de densité au point $B$, par suite du balayage d'une masse $m$ située en $A$, à cette masse $m$. Nous avons ainsi une limite superieure de $\frac{\Sigma \delta}{\Sigma m}$, et comme $\Sigma m$ est au plus égale à I, nous avons une limite $H$ de $\Sigma \delta$, c'est à dire de l'accroissement total de la densité en $B$ par suite du balayage de $C_{k}$. Si nous supposons qu'entre deux balayages consécutifs de $C_{k}$, on balaye toujours l'un des éléments dont fait partie $B$, nous voyons qu'avant chaque balayage de $C_{k}$, la densité en $B$ est nulle; après ce balayage elle prend une valeur inférieure à $H$, (limite de $\Sigma \delta$ ) et la conserve jusqu'à ce qu'on balaye un des éléments dont fait partie $B$, elle redevient alors nulle. La densité au point $B$ est donc limitée.

Je dis maintenant que les dérivées $\frac{d u_{n}}{d \xi}, \frac{d u_{n}}{d \eta}$ sont limitées au point $B$. Décrivons en effet autour du point $B$ un petit cercle $K$. Soit $\varphi_{n}$ le potentiel logarithmique dû à l'attraction des masses situées à l'intérieur de $K$ et qui sont toutes sur la circonférence de $C_{k}$; les densités étant limitées, $\varphi_{n}$ et ses dérivées seront limitées; la fonction $u_{n}$ qui est plus petite que $u$ est limitée. La différence $u_{n}-\varphi_{n}$ est donc limitée à l'intérieur de $K$ et sur la circonférence; de plus cette différence $u_{n}-\varphi_{n}$ est harmonique à l'intérieur de $K$; nous pouvons done par l'application du théorème de HARNACK, trouver une limite supérieure des dérivées de $u_{n}-\varphi_{n}$, et par conséquent de celles de $u_{n}$, puisque celles de $\varphi_{n}$ sont limitées.

C. Q. F. D.

Ce qui précède ne s'appliquerait pas à un point $B$ qui serait à l'intersection de deux lignes de discontinuité; ou verrait en effet qu'en un pareil point, la densité pourrait devenir logarithmiquement infinie, (je veux dire que la densité en un point $B^{\prime}$ de $C_{k}$, très voisin de $B$ est de l'ordre de logarithme de la distance $B B^{\prime}$.) Il en est de même pour les dérivées de $u_{n}$. Au contraire la fonction $u_{n}$ elle-même, toujours plus petite que $u$, reste finie.

Mais nous pouvons toujours éviter de faire passer nos chemins d'intégration par ces points d'intersections des lignes de discontinuité. Le résultat resterait le 
même d'ailleurs si on les y faisait passer, car bien que $\frac{d u_{n}}{d \xi}$ devienne logarithmiquement infinie, l'intégrale $\int \frac{d u_{n}}{d \xi} d \eta$ resterait finie. Il résulte de là que l'intégrale

$$
\int\left(d v-d v_{n}\right)
$$

prise le long d'un chemin quelconque tend vers zéro quand $n$ croît indéfiniment.

On a vu que les fonctions $z_{n}$ ne sont définies qu'à un facteur constant près de module I qui peut varier brusquement quand on franchit une des lignes $L$, ou ce qui revient au même que les fonctions $v_{n}$ ne sont définies qu'à une constante près qui varie brusquement quand on franchit une des lignes $L$. Les fonctions $v_{n}$ outre les variations continues:

$$
\frac{d u_{n}}{d \xi} d \eta-\frac{d u_{n}}{d \eta} d \xi
$$

qu'elles subissent quand on décrit un chemin quelconque, subissent donc des variations brusques quand on franchit une ligne $L$.

On peut pour achever de définir les $v_{n}$, convenir que ces variations brusques en certains points de certaines lignes $L$, doivent être nulles. Mais ces variations ne peuvent être nulles partout. Envisageons en effet les intégrales

$$
\int d v, \int d v_{n}
$$

prises le long d'un chemin fermé quelconque. Elles sont égales à $2 \pi$ multiplié par la somme des masses intérieures au contour d'intégration. Pour $v$, il ne peut y avoir que la masse $+\mathrm{I}$ située au point $O$; pour $v_{n}$ il peut y avoir en outre des masses négatives sur les lignes $L$. Si donc le contour enveloppe le point $O$, la $\mathrm{I}^{\text {ere }}$ intégrale sera égale à $2 \pi$ et la $2^{\text {de }}$ généralement plus petite que $2 \pi$; si le contour n'enveloppe pas le point $O$, la $1^{\text {ere }}$ intégrale sera égale à $O$ et la $2^{\text {de }}$ généralement négative.

\section{§ 6. Les fonctions $\boldsymbol{u}_{p \cdot n}$.}

Nous avons dit que la fonction $u$ obtenue était indépendante de l'ordre des balayages; mais nous avons jusqu'ici supposé que ceux-ci étaient dirigés de telle façon que l'on pouvait trouver une fonction $u_{n}$ dont l'indice $n$ est un entier fini et pour laquelle un élément quelconque aurait été balayé un nombre de fois aussi grand qu'on le veut. Nous considérions alors la série 


$$
u=u_{0}+\left(u_{1}-u_{0}\right)+\cdots+\left(u_{n+1}-u_{n}\right)+\cdots
$$

comme une série ordinaire; nous pouvons aussi la traiter comme une série à double entrée.

Supposons une suite de fonctions d̀ deux indices $u_{n p}$. La I ${ }^{\text {ere }} u_{00}$ sera celle que nous appelions jusqu'ici $u_{0} ; u_{0 . n+1}$ se déduira de $u_{0 . n}$ par le balayage d'un certain élément $C_{k} ; u_{10}$ sera la limite de $u_{0 . n}$ pour $n=\infty ; u_{1 . n+1}$ se déduira de $u_{1 . n}$ par un balayage; $u_{20}$ sera la limite de $u_{1 . n}$ pour $n=\infty$; et ainsi de suite; $u_{p . n+1}$ se déduira de $u_{p . n}$ par un balayage, et $u_{p+1.0}$ sera la limite de $u_{p . n}$ pour $n=\infty$. Il est clair que l'on anra:

$$
u_{p+1 . m}>u_{p+1.0}>u_{p . n+1}>u_{p . n}>u_{p .0}>\cdots>u_{00}>0,
$$

On verrait comme plus haut que

$$
u>u_{p . n}
$$

ce qui montrerait que la suite des $u_{p . n}$ quand $n$ puis $p$ tendent vers l' $\infty$ a une limite $\leqq u$; et comme cette limite est une fonction harmonique en vertu du théorème de HARNACK, elle est plus grande que les $u_{n}$ que nous avions envisagés dans les $\$$ précédents. Elle est donc $\geqq u$; elle est donc égale à $u$; c'est toujours le raisonnement de la fin du $\$ 3$ qui s'applique sans changement.

Nous pouvons par exemple, considérer une série de domaines

$$
d_{0}, d_{1}, d_{2}, \ldots
$$

contenus dans notre domaine $D$, et tels que chacun d'eux soit contenu dans le suivant. De plus chacun d'eux sera formé d'un nombre fini d'éléments de $D$. Alors nous supposerons que les balayages qui font passer de $u_{0 n}$ à $u_{0 n+1}$ ne portent que sur les éléments de $d_{0}$ et de telle façon que chacun d'eux soit balayé une infinité de fois; que les balayages qui font passer de $u_{1 . n}$ à $u_{1, n+1}$ ne portent que sur les éléments de $d_{1}$ et cela de telle façon que chacun d'eux soit balayé une infinité de. fois; et ainsi de suite.

Dans ces conditions, il est clair que $u_{1.0}$ est harmonique dans tout le domaine $d_{0}$, et nul (de même que toutes les fonctions $u_{0 . n}$ ) en dehors de ce domaine et sur ses frontières; que $u_{2.0}$ est harmonique dans tout le domaine $d_{1}$ et nul (de même que toutes les fonctions $u_{1 . n}$ ) en dehors de ce domaine $d_{1}$ et sur ses frontières et ainsi de suite.

Toutes les conclusions des $\$$ précédents s'appliquent sans changement; on peut définir les fonctions $v_{n p}$ et $z_{n p}$ à l'aide des $u_{n p}$ comme on a défini les $v_{n}$ et les $z_{n}$ à l'aide des $u_{n}$. On pourrait seulement se demander si l'on peut encore comme au $\$$ précédent limiter la densité des masses génératrices en un point $B$, et si les conséquences déduites de cette limitation subsistent. Il faut pour cela, 
comme nous l'avons dit, que (si $B$ est sur la circonférence de $C_{k}$ ) entre deux balayages consécutifs de $C_{k}$, on balaye toujours l'un des éléments dont fait partie $B$. Il est clair qu'on peut remplir cette condition pour les fonctions $u_{0 n}$ si $B$ est à l'intérieur du domaine $d_{0}$ et non pás sur sa frontière; pour les fonctions $u_{1 . n}$ si $B$ est à l'intérieur du domaine $d_{1}$ et non par sur sa frontière, et ainsi de suite. Quelle que soit donc la position de $B$, on pourra prendre $p$ assez grand, pour que la condition ne cesse plus d'être remplie à partir du moment où on aura formé la fonction $u_{p, 1}$. Nous pouvons donc raisonner comme au $\S$ précédent et conclure que

$$
\int\left(d v-d v_{p n}\right)
$$

prise le long d'un chemin quelconque, tend vers zéro, quand on fait tendre $n$ puis $p$ vers l'infini.

Dans le mémoire cité (Bull. Soc. Math.) nous avons envisagé seulement les fonctions

$$
u_{1.0}, u_{2.0}, \ldots, u_{p .0} \ldots
$$

qui sont les fonctions de GREEN relatives aux domaines successifs

$$
d_{1}, d_{2}, \ldots, d_{p} \ldots
$$

Nous y trouvions l'avantage que la fonction $u_{p .0}$ est harmonique à l'intérieur du domaine $d_{p}$, de sorte que la fonction $z_{p .0}$ est uniforme et continue dans le domaine $d_{p}$, (ce qui n'arrive pas pour les fonctions $z_{n}$ susceptibles comme nous l'avons vu de variations brusques.) Nous évitions ainsi en partie la discussion du $\S$ précédent; j'ai cru pourtant que cette discussion était par elle-même instructive et je n'ai pas voulu pour cette raison me servir ici du même artifice.

\section{§ 7. Propriétés de la fonction $\approx$.}

Faisons maintenant varier la position du point $O$; et amenons-le en $O^{\prime}$; (le

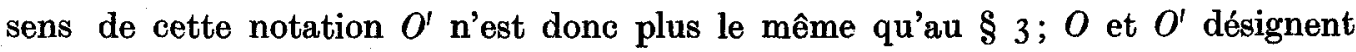
simplement deux points quelconques du domaine $D$.) On peut former deux fonctions de GREEN $u$ et $u^{\prime}$ devenant logarithmiquement infinies, l'une en $O$, l'autre en $O^{\prime}$, et ce sont ces deux fonctions que je me propose de comparer.

Nous pourrons diriger les balayages soit comme au $\S 3$, soit comme au $\S 6$; nous aurons dans le premier cas une suite de fonctions $u_{n}$, dans le second cas une suite de fonctions à double indice $u_{p n}$; mais ces deux suites ont même limite. Nous désignerons par $u_{n}^{\prime}, u_{p n}^{\prime}, v_{n}^{\prime}, v_{p n}^{\prime}, z_{n}^{\prime}, z_{p n}^{\prime}$, les fonctions qui sont à $u^{\prime}$ ce que $u_{n}, u_{p n}, v_{n}, v_{p n}, z_{n}, z_{p n}$ sont à $u$. 
Considérons en particulier les fonctions $z_{p .0}, z_{p .0}^{\prime}$; soit $a_{p}$ la valeur de $z_{p .0}$ en $O^{\prime}$ et $a_{p}^{\prime}$ celle de $z_{p .0}^{\prime}$ en $O$. Je dis que $z_{p .0}$ ne pourra prendre qu'une seule fois la valeur $a_{p}$; prenons en effet l'intégrale:

$$
\int \frac{d z_{p .0}}{z_{p .0}-a_{p}}
$$

le long de la frontière du domaine $d_{p-1}$; elle est égale à $2 i \pi$; en effet quand nous ferons le tour de cette frontière, le point $z_{p .0}$ décrira un cercle de rayon I puisque $u_{p .0}$ est nul sur toute cette frontière, et il le décrira une fois et une seule. En effet $\int d v_{p .0}$ est égal à $2 \pi$, puisqu'en ce qui concerne cette fonction $u_{p .0}$ il n'y a à l'intérieur du domaine $d_{p-1}$ d'autre masse génératrice qu'une masse + I au point $O$. Le point $a_{p}$ est d'ailleurs à l'intérieur du cercle de rayon I décrit par le point $z_{p .0}$; l'intégrale (I) est égale à $2 i \pi$. Or cette intégrale est égale à $2 i \pi$ multipliée par le nombre de fois que la fonction $z_{p .0}$ prend la valeur $a_{p}$ à l'intérieur de $d_{p-1}$. Donc la fonction ne prend qu'une seule fois cette valeur.

C. Q. F.'D.

Cette démonstration ne peut donner lieu ici à aucune objection, car le domaine $d_{p-1}$ se composant d'un nombre fini d'éléments, sa frontière se compose d'un nombre fini d'arcs de cercle; la fonction $u_{p .0}$ peut être ainsi définie, comme l'a montré M. Schwarz, à l'aide d'une équation différentielle linéaire à coëfficients rationnels, de la même façon que les fonctions fuchsiennes. On pourrait d'ailleurs appliquer l'un quelconque des procédés pour la démonstration complète du principe de Derichlet quand la frontière du domaine envisagé est formée de lignes analytiques, ou plus généralement quand elle a un rayon de courbure déterminé. (Cf. loco cit. American Journal pages 227, 228, 229.) Il n'y aurait rien à y changer.

Nous sommes donc certains que $u_{p .0}$ tend bien vers 0 quand on se rapproche indéfiniment de la frontière de $d_{p-1}$; et on peut appliquer les résultats obtenus plus haut, non seulement à l'intérieur, mais à la frontière de $d_{p-1}$. On démontrerait de la même manière que $z_{p .0}^{\prime}$ ne peut prendre qu'une seule fois la valeur $a_{p}^{\prime}$.

Cela posé soient $p_{p}$ et $\varphi_{p}$ le module et l'argument de $a_{p}$, envisageons le rapport:

$$
R=z_{p .0}^{\prime} \frac{p_{p} z_{p .0}-e^{i \varphi p}}{z_{p .0}-p_{p} e^{i \varphi p}}
$$

Je dis que ce rapport ne peut, à l'intérieur de $d_{p-1}$, devenir ni nul, ni infini; en effet $z_{p, 0}^{\prime}$ et $z_{p .0}$ étant finis à l'intérieur de ce domaine, $R$ ne pourrait devenir nul ou infini, que si l'on avait: 
$I^{0}$ ou bien $z_{p, 0}^{\prime}=0$ ce qui ne peut arriver qu'au point $O^{\prime}$.

$2^{\circ}$ ou bien $\rho_{p} z_{p .0}-e^{i \varphi p}=0$, ce qui est impossible puisque $\rho_{p}<\mathrm{I},\left|z_{p .0}\right|<\mathrm{I}$, $\left|e^{i \varphi p}\right|=\mathbf{I}$.

$3^{\circ}$ ou bien $z_{p .0}-p_{p} e^{i \varphi p}=0$, ou $z_{p .0}=a_{p}$; ce qui a lieu en $O^{\prime}$ par l'hypothèse, et ne peut avoir lieu en aucun autre point par suite du théorème que nous venons de démontrer.

Il n'y aurait donc que pour le point $O^{\prime}$, et en ce point le numérateur et le dénominateur s'annulent et la fraction reste finie.

Donc $\log |R|$ est une fonction harmonique à l'intérieur de $d_{p-1}$.

De plus quand on décrit la frontière de $d_{p-1}$, on a

$$
\left|z_{p .0}\right|=\left|z_{p .0}^{\prime}\right|=|R|=\mathrm{I}
$$

d'où $\log |R|=0$; cette fonction s'annulant à la frontière du domaine et étant harmonique dans tout le domaine, doit s'annuler dans tout le domaine. Done $R$ est une constante dont le module est égal $\grave{a} \mathrm{I}$.

Pour déterminer cette constante je donnerai aux fonctions leurs valeurs au point $O$, c'est à dire:

$$
z_{p, 0}^{\prime}=a_{p}^{\prime}, z_{p .0}=0, R=\frac{a_{p}^{\prime}}{\rho_{p}} .
$$

On a donc entre $z_{p .0}^{i}$ et $z_{p: 0}$ la relation linéaire:

$$
z_{p .0}^{\prime}=\frac{a_{p}^{\prime}}{\rho_{p}} \frac{z_{p .0}-\rho_{p} e^{i \varphi p}}{\rho_{p} z_{p .0}-e^{i \varphi p}}
$$

Soit maintenant

$$
a=p e^{i p}
$$

la valeur de $z$ au point $O^{\prime}$, et $a^{\prime}$ la valeur de $z^{\prime}$ au point $O$. Faisons croître $p$ indéfiniment, $z_{p .0}, z_{p .0}^{\prime}, a_{p}, \rho_{p}, \varphi_{p}, a_{p}^{\prime}$ tendront vers $z, z^{\prime}, a, \rho, \stackrel{\varphi}{p}, a^{\prime}$, et à la limite la relation (2) devient

$$
z^{\prime}=\frac{a^{\prime}}{\rho} \frac{z-\rho e^{i \varphi}}{\rho z-e^{i \varphi}} .
$$

Donc les deux fonctions $z$ et $z^{\prime}$ sont liées par une relation linéaire. Ce n'est pas tout; $z^{\prime}$ ne peut s'annuler qu'une fois; à savoir au point $O^{\prime}$. Donc $z$ ne peut prendre qu'une fois la valeur $a$, c'est à dire la valeur qu'il prend au point $O^{\prime}$; et comme $O^{\prime}$ est quelconque, la fonction $z$ ne' peut prendre qu'une seule fois la même valeur. 


\section{§ 8. Représentation conforme du domaine $D$ sur un cercle.}

Résumons les résultats obtenus; nous avons $v u$ qu'il existe une fonction analytique $z$ du domaine $D$, qui ne peut prendre que des valeurs de module plus petit que I, et qui ne peut prendre chacune de ces valeurs qu'une seule fois, mais nous ne savons pas encore si elle peut prendre toutes ces valeurs. En d'autres termes, traçons dans le plan des $z$ le cercle $K$, de centre $O$ et de rayon I. Nous aurons une représentation conforme du cercle $K$ sur le domaine $D$, de telle façon qu'à tout point de $D$ corresponde un point de $K$ et un seul, et qu'à tout point de $K$ ne puisse correspondre plus d'un point de $D$. Mais nous ne savons pas encore si à tout point de $K$ correspond un point de $D$. C'est ce dernier point qu'il s'agit d'établir.

Nous serons donc amenés à distinguer dans $K$ deux ensembles de points: ${ }^{\circ}{ }^{0}$ l'ensemble $E$ des points de $K$ auxquels correspond un point de $D .2^{\circ}$ l'en- $^{\text {'en }}$ semble $E_{1}$ des points de $K$ auxquels ne correspond aucun point de $D$. Je me propose de démontrer que l'ensemble $E$ comprend tout l'intérieur de $K$ et que l'ensemble $E_{1}$ se réduit à la circonférence de $K$.

Observons d'abord que autour de tout point de $E$ on peut décrire un cercle de rayon assez petit pour que tous les points intérieurs à ce cercle appartiennent également à $E$, et en effet la fonction $z$ étant analytique, à toute aire de $D$ correspondra une aire de $K$. L'ensemble $E$ ne contient donc pas de points isolés, et est contenu dans son propre dérivé $E^{\prime}$. En revanche $E^{\prime}$ contiendra des points qui n'appartiendront pas à $E$ et qui représenteront la frontière de $E$.

Aux différents éléments $C_{k}$ de $D$ correspondront des aires $\gamma_{k}$ du cercle $K$; toutes ces aires feront partie de $E$. Deux de ces aires ne pourront avoir de points communs que si elles correspondent à deux éléments contigus de $D$, c'est à dire à deux éléments tels que certains points de l'un soient identiques à certains points de l'autre puisqu'à un point de $K$ ne peut correspondre qu'un seul point de $D$ (Cf. $\S 2$.) Je remarque d'autre part que l'ensemble $E$ est simplement connexe, je veux dire par là que si une courbe fermée fait partie de $E$, tous les points intérieurs à cette courbe feront également partie de $E$. Cela résulte de ce fait que le domaine $D$ est lui-même simplement connexe.

Si alors tous les points d'une courbe fermée $C$ font partie de $E$, c'est à dire ont leurs images sur $D$, l'ensemble de ces images formeront aussi une courbe fermée. $D$ étant simplement connexe, cette courbe enfermera une aire faisant partie de $D$. Les images sur $K$ des différents points de cette aire, formeront à leur tour une aire qui ne sera autre chose que l'aire limitée par $C$. 
Si $C$ est une circonférence de centre $O$, il n'y aura à l'intérieur de cette circonférence aucun point de $E_{1}$; si done il n'y a pas de points de $E_{1}$ dont la distance à $O$ soit égale à $a$, il n'y en aura pas non plus dont la distance soit plus petite que $a$.

Cela posé, nous allons donner de notre proposition une première démonstration fondée sur la méthode de OsGood.

Soit $q$ un point quelconque de la frontière de $E$, soient $q_{1}$ et $q_{2}$ deux autres points de $E_{1}$ d'ailleurs quelconques. Construisons une fonction fuchsienne:

$$
\varphi(w)
$$

définie de la façon suivante: $I^{0}$ elle ne devra prendre aucune des trois valeurs

$$
q, q_{1}, q_{2} \text {. }
$$

$2^{\circ}$ le polygone générateur aura tous ses sommets sur le cercle fondamental (c'est à dire qu'il sera de la $3^{\circ}$ famille); ce sera un quadrilatère dont les côtés seront conjugués deux à deux, de façon que deux côtés conjuguées soient adjacénts (c'est à dire qu'il sera de genre o); quant $w$ se rapprochera de l'un des quatre sommets, $\varphi(w)$ tendra vers respectivement $q, q_{1}, q_{2}$ ou $q_{1}$, (de sorte que notre fonction $\varphi(w)$ sera liée à la fonction modulaire par une relation linéaire simple.) $3^{0}$ de plus on aura

Posons alors:

$$
\varphi(0)=0
$$

$$
z=\varphi(w)
$$

on voit que $w$ est une fonction multiforme de $z$ et qui ne peut prendre que des valeurs dont le module est plus petit que r. Quand $z$ tend vers $q$ le module de toutes les déterminations de $w$ tend vers l'unité, je précise: si parmi toutes les déterminations de $w$, celle dont le module est le plus petit est $w_{0}$, le module $\left|w_{0}\right|$ est plus grand qu'une fonction $\theta(|z-q|)$ qui tend vers l'unité quand $|z-q|$ tend vers zéro.

J'observe de plus qu'à l'intérieur de $\boldsymbol{E}$ la fonction $w$ peut être regardée comme uniforme. En effet, cet ensemble $\boldsymbol{E}$ étant simplement connexe, une courbe fermée tracée sur $E$ ne peut contenir à son intérieur que des points de $E$; elle ne peut donc contenir aucun des 3 points singuliers $q, q_{1}, q_{2}$. Nous choisirons la détermination de $w$ qui s'annule pour $z=0$.

Posons alors

$$
t=\log \left|\frac{x}{w}\right|
$$

cette fonction $t$ sera harmonique dans $E$ sauf en $O$ où elle devient logarithmiquement infinie; de plus elle est toujours positive. 
Comparons-la à une des fonctions $u_{n}$; ces fonetions, harmoniques dans $D$, le seront aussi sur $K$, puisque la représentation de $D$ sur $K$ est conforme. Il n'y aura d'exception que pour le point $O$ où ces fonctions deviendront logarithmiquement infinies et pour les lignes correspondant aux lignes de discontinuité $L$ des $§$ précédents et qui en sont l'image sur $K$; dans le voisinage de ces lignes que nous appellerons $l$, les fonctions $u_{n}$ se comportent comme des potentiels logarithmiques engendrés par des masses négatives répandues sur ces lignes.

La différence $t-u_{n}$ sera donc une fonction harmonique, (même au point $O$ ) sauf sur les lignes $l$ où elle se comportera comme un potentiel logarithmique engendré par des masses positives répandues sur ces lignes; elle pourra donc avoir des maxima, mais pas de minima.

Sois alors $e_{n}$ l'ensemble des points appartenant aux divers éléments que l'on a dû successivement balayer pour obtenir $u_{n}$; sur la frontière de $e_{n}$ et en dehors de $e_{n}$, on a:

$$
t>0, u_{n}=0, t-u_{n}>0
$$

on aura donc aussi à l'intérieur de $e_{n}$ (où il ne peut pas y avoir de minima):

$$
t-u_{n}>0 \quad t>u_{n}
$$

et comme cela a lieu quel que soit $n$, on a dans $E$, ̀̀ la limite.

$$
t \geqq u \text {. }
$$

Mais nous pouvons trouver une suite de points

$$
m_{1}, m_{2}, \ldots, m_{h}, \ldots
$$

qui appartiennent à $E$ et qui se rapprochent indéfiniment de $q$, puisque $q$ fait partie de $E^{\prime}$. Si

$$
\lim m_{h}=q, \quad \lim \theta\left(\left|m_{h}-q\right|\right)=\mathrm{I} .
$$

Soient $t_{h}$ et $w_{h}$ les valeurs de $t$ et de $w$ correspondant à $m_{h}$, on aura

$$
\left|w_{h}\right|>\theta\left(\left|m_{h}-q\right|\right), \quad \lim \left|w_{h}\right|=\mathrm{r}, \quad \lim t_{h}=0 .
$$

Or $u=\log \left|\frac{\mathrm{I}}{z}\right|$ et se réduit à $\log \left|\frac{\mathrm{I}}{m_{h}}\right|$ pour $z=m_{k}$. L'inégalité $t>u$ nous donne donc

On a donc

$$
t_{h}>\log \left|\frac{\mathrm{I}}{m_{h}}\right|>0 \text {. }
$$

$$
\lim \log \left|\frac{1}{m_{h}}\right|=0
$$


et à la limite

$$
\log \left|\frac{\mathrm{I}}{q}\right|=0, \quad|q|=\mathbf{I}
$$

Le point $q$ est donc sur la circonférence du cercle $K$, et comme $q$ est quelconque, tous les points de la frontière sont sur cette circonférence, c'est à dire que $\boldsymbol{E}$ comprend tous les points intérieurs à $\boldsymbol{K}$.

C. Q. F. D.

Nous allons donner de la même proposition une seconde démonstration.

Remarquons d'abord que les $u_{n}$ considérées comme fonctions de $z$ vont se comporter comme des potentiels logarithmiques; et les masses génératrices seront les mêmes que les masses correspondantes, quand on regardait les $u_{n}$ comme fonctions de $x=\xi+i \eta$. Je ne veux pas dire que les densités restent les mêmes, parce que les aires et les longueurs sur $K$ ne sont pas égales aux aires et aux longueurs sur $D$; mais dans deux aires, ou dans deux arcs correspondants, les quantités totales de matière attirante sont les mêmes. Je fais en passant cette remarque bien qu'elle ne doive jouer aucun rôle essentiel dans la démonstration qui va suivre, parce qu'elle découle immédiatement des propriétés de la représentation conforme.

Nous allons nous appuyer sur l'un des théorèmes de Grews, qui est le suivant. Soient $V$ et $V^{\prime}$ deux potentiels par exemple logarithmiques, on aura l'identité:

$$
\sum m V^{\prime}=\sum m^{\prime} V
$$

les masses $m$ sont celles qui engendrent le potentiel $V$, et chacune d'elles doit être multipliée par la valeur de $V^{\prime}$ au point occupé par cette masse. De même les masses $m^{\prime}$ sont celles qui engendrent $V^{\prime}$, chacune d'elles doit être multipliée par la valeur correspondante de $V$.

Nous allons appliquer ce théorème:

$I^{\circ}$. A la fonction $u$ regardée comme fonction de $z$, c'est un potentiel logarithmique engendré par une masse + I située au point o, et par des masses négatives $-m$ réparties sur la circonférence de $K$.

$2^{\circ}$. A la fonction $u_{n}$ regardée comme fonction de $z$, c'est un potentiel logarithmique engendré par une masse + I située au point $o$, et par des masses négatives $-m_{n}$ réparties sur les lignes de discontinuité $l$.

3o. A un potentiel auxiliaire $w$ que nous allons définir.

Soit

$$
|z|=r=e^{-u}
$$

Soit $W$ un potentiel logarithmique, dû à des masses toutes situées à l'intérieur de $K$ et au sujet desquelles je suppose: 
Io que la somme des masses $\mu$ contenues entre les deux circonférences concentriques à $K$ et de rayons $r^{\prime}$ et $r^{\prime}+d r^{\prime}$, que cette somme, dis-je, est égale à $h^{\prime} d r^{\prime}, h^{\prime}$ étant une fonction quelconque de $r^{\prime}$, positive et finie.

$2^{\circ}$ que si sur la circonférence de rayon $r^{\prime}$, il y a des points de $E_{1}$, toutes les masses $\mu$ qui se trouvent sur cette circonférence, sont sur $E_{1}$.

Si alors $r^{\prime}$ et $\theta^{\prime}$ sont les coordonnées polaires de la masse attirante $\mu ;$ si $r=e^{-u}, \theta=-v$ sont celles du point attiré, on aura:

$$
W=\sum \mu \log \frac{\mathbf{I}}{\sqrt{r^{2}+r^{2}-2 r r^{\prime} \cos \left(\theta-\theta^{\prime}\right)}}
$$

Soit $W^{\prime}$ le potentiel que l'on déduit de $W$ en balayant le cercle $K$; il est dû à des masses positives $\mu^{\prime}$ situées sur la circonférence de $K$, et il est égal à $W$ en dehors de $K$ et sur la circonférence de $K$. Posons enfin

$$
w=W-W^{\prime}
$$

La potentiel $w$ est alors dû aux masses $\mu$ et $-\mu^{\prime}$; il est égal à zéro hors de $K$ et sur la circonférence de $K$. A l'intérieur de $K$ on a:

$$
w=\sum \mu \log \sqrt{\frac{r^{2} r^{\prime 2}+1-2 r r^{\prime} \cos \left(\theta-\theta^{\prime}\right)}{r^{2}+r^{\prime 2}-2 r r^{\prime} \cos \left(\theta-\theta^{\prime}\right)}}>0 .
$$

Le radical du second membre, quand on fait varier $\theta-\theta^{\prime}$, atteint son maximum pour $\theta-\theta^{\prime}=0$ et cela quels que soient $r$ et $r^{\prime}$ (qui sont $<1$ ). On a donc:

$$
w<\sum \mu \log \frac{\mathrm{I}-r r^{\prime}}{\left|r-r^{\prime}\right|}=\int_{0}^{1} h^{\prime} d r^{\prime} \log \frac{\mathrm{I}-r r^{\prime}}{\left|r-r^{\prime}\right|} .
$$

On voit aisément que le dernier membre est toujours fini et positif; qu'il s'annule pour $r=1$ et que si $h^{\prime}$ s'annule pour $r^{\prime}=1$, si par exemple $h^{\prime}=\mathrm{I}-r^{\prime}$, il s'annule comme $u$ de sorte que le rapport de $w$ à $u$ reste fini. On peut donc trouver une quantité positive $\lambda$ telle que

$$
w<\lambda u \text {. }
$$

La formule (I) appliquée à $u$ et à $u_{n}$ nous donne:

$$
\left(u-u_{n}\right)_{0}=\sum m_{n} u-\sum m u_{n}
$$

Le I $^{\text {er }}$ membre représente la valeur de $u-u_{n}$ au point $O$ où sont les deux masses +1 ; dans le second membre, il faut donner à $u$ les valeurs correspondant aux diverses masses $m_{n}$ et à $u_{n}$ les valeurs correspondant aux diverses masses $m$. 
Mais ces dernières masses sont toutes sur $K$ et sur cette circonférence $u_{n}$ est nul. Nous pouvons done écrire:

$$
\left(u-u_{n}\right)_{0}=\sum m_{n} u .
$$

Appliquons maintenant le théorème de GREEN à $u$ et à $w$, il viendra:

$$
w_{0}-\sum m w=\sum u-\Sigma \mu^{\prime} u,
$$

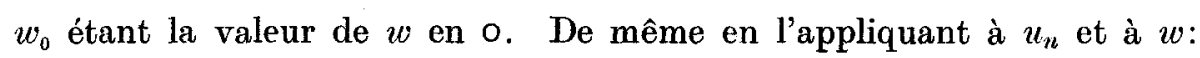

$$
w_{0}-\Sigma^{\prime} m_{n} w=\sum \mu u_{n}-\sum \mu^{\prime} u_{n} .
$$

Toutes les masses $\mu^{\prime}$ sont sur la circonférence $K$ de même que toutes les masses $m$, or sur $K$ on a:

$$
w=u=u_{n}=0 \text {. }
$$

Nos équations peuvent donc s'écrire:

$$
\begin{aligned}
& w_{0}=\sum \mu \cdot u \\
& w_{0}-\sum m_{n} w=\sum \mu \cdot u_{n}
\end{aligned}
$$

ou en retranchant, et tenant compte de (2)

$$
\sum \mu\left(u-u_{n}\right)=\sum m_{n} w<\lambda \sum m_{n} u
$$

ou en vertu de (3)

$$
\sum \mu\left(u-u_{n}\right)<\lambda\left(u-u_{n}\right)_{0}
$$

la différence $u-u_{n}$ est toujours positive; mais sur les points de $E_{1}$ on a $u_{n}=0$; de sorte que si sur la circonférence de rayon $r^{\prime}$, il y a des points de $E_{1}$, comme toutes les masses $\mu$ de cette circonférence seront en ces points, la valeur correspondante de $u-u_{n}$ sera:

On aura done

$$
u=\log \frac{\mathrm{I}}{r^{\prime}}
$$

$$
\sum \mu\left(u-u_{n}\right)>\int_{\alpha}^{1} h^{\prime} d r^{\prime} \log \frac{\mathrm{I}}{r^{\prime}} .
$$

a étant la plus courte distance du point $O$ à l'ensemble $E_{1}$; car s'il y a des points de $E_{1}$ pour $r^{\prime}=\alpha$, il y en aura, comme nous l'avons montré plus haut pour $r^{\prime}>\alpha$. On a donc

$$
\int_{\alpha}^{1} h^{\prime} d r^{\prime} \log \frac{\mathrm{I}}{r^{\prime}}<\lambda\left(u-u_{n}\right)_{0} .
$$


Quand $n$ croît indéfinement la différence $\left(u-u_{n}\right)_{0}$ tend vers zéro; il en est done de même du ${ }^{\text {er }}$ membre, ce qui veut dire que $x$. tend vers $I$; on a donc

$$
\alpha=1 \text {, }
$$

ce qui veut dire que l'ensemble $E_{1}$ se réduit à la circonférence $K$.

C. Q. F. D.

\section{§ 9. Propriétés des fonctions uniformisantes.}

Considérons un système quelconque

$y_{1}, y_{2}, \ldots$

de fonctions multiformes de $x$. Nous avons vu au $\$ 4$ que si $D$ est le domaine principal de ce système, on peut toujours trouver un domaine $D^{\prime}$ régulièrement multiple de $D$, et simplement connexe, pour lequel la fonction de GreEn $u$ existe.

Ce domaine $D^{\prime}$ étant simplement connexe, les $\$ 5$ et 8 nous apprendront qu'on peut déduire de $u$ une fonction $z$; que si dans le plan des $z$ on trace le cercle $K$ de centre $O$ et de rayon I; à tout point de $K$ correspond un point de $D^{\prime}$ et un seul, et réciproquement. Donc

$$
y_{1}, y_{2}, \ldots, x
$$

sont des fonctions uniformes de $z, x=\psi(z), y_{i}=\theta_{i}(z)$.

J'ajoute qu'à tout point pour lequel les fonctions $y_{1}, y_{2}, \ldots$, existent toutes, correspondra un point intérieur à $K$, et non pas un point situé sur la circonférence de $K$. Il suffit pour obtenir ce résultat de choisir convenablement la fonction fuchsienne auxiliaire du $\$ 4$ et d'admettre les éléments algébriques. Il n'y a pas d'Ausnahmsstellen.

Cherchons maintenant les propriétés de ces fonctions uniformes de $z$,

$$
x=\psi(z), y_{i}=\theta_{i}(z) .
$$

Nous avons dit que $D^{\prime}$ était régulièrement multiple de $D$; cela veut dire qu'à tout point $M$ de $D$; correspondent divers points

$$
M_{1}, M_{2}, \ldots
$$

de $D^{\prime}$; si de plus le point $M$ varie sur $D$ d'une manière continue, il en sera de même des points $M_{1}, M_{2}, \ldots$, sur $D^{\prime}$. Si $M$ décrit une courbe fermée infiniment petite, il en sera de même de $M_{1}, M_{2}, \ldots$, mais cela ne sera plus vrai en général si la courbe fermée décrite par $M$ n'est pas infiniment petite.

Je dis maintenant que si $M_{1}$ décrit une courbe fermée finie, il en sera de même de $M_{2}$. Cela tient à ce que le domaine $D^{\prime}$ est simplement connexe. Soit en effet $C$ la courbe fermée décrite par $M_{1}$, elle limitera une certaine aire située 
sur $D^{\prime}$. Décomposons cette aire en une infinité d'ajres infiniment petites. Quand $M_{1}$ décrira le contour d'une de ces aires partielles, $M_{2}$ reviendra à sa valeur initiale et décrira une courbe fermée. Il devra donc en être de même quand $M$; décrira le contour de l'aire totale.

Donc $M_{2}$ est fonction uniforme de $M_{1}$ et réciproquement. Soit

$$
M_{2}=\Omega\left(M_{1}\right)
$$

la fonction ainsi définie; si $A$ est une courbe, ou une aire décrite par le point $M_{1}$ alors $\Omega(A)$ sera la courbe ou l'aire correspondante décrite par $M_{2}$. Par exemple cette aire $A$ peut-être un des éléments du domaine $D^{\prime}$ et alors $\Omega(A)$ sera un autre élément de $D^{\prime}$.

Cela posé considérons les fonctions

$$
u_{0}, u_{1}, u_{2}, \ldots, u_{n}, \ldots
$$

formées en partant du point $O$ et en balayant successivement les éléments

du domaine $D^{\prime}$.

$$
C_{1}, C_{2}, \ldots, C_{n}, \ldots
$$

Formons ensuite les fonctions

$$
u_{0}^{\prime}, u_{1}^{\prime}, u_{2}^{\prime}, \ldots, u_{n}^{\prime}, \ldots
$$

en partant du point $\Omega(0)$ et en balayant successivement les éléments

du domaine $D^{\prime}$.

$$
\Omega\left(C_{1}\right), \Omega\left(C_{2}\right), \ldots, \Omega\left(C_{n}\right), \ldots
$$

Les éléments correspondants $C_{n}$, et $\Omega\left(C_{n}\right)$ coincident an point de vue géométrique, de même que les points $O$ et $\Omega(O)$; les opérations donneront done les mêmes résultats et on aura, pour un point $M_{1}$ quelconque du domaine $D^{\prime}$

et à la limite:

$$
u_{n}^{\prime}\left[\Omega\left(M_{1}\right)\right]=u_{n}\left[M_{1}\right]
$$

$$
u^{\prime}\left[\Omega\left(M_{1}\right)\right]=u\left[M_{1}\right]
$$

en appelant $u^{\prime}$ et $u$ les limites des deux suites $u_{n}^{\prime}$ et $u_{n}$.

Or $u^{\prime}$ et $u$ sont les fonctons de GREen formées respectivement en partant des deux points $\Omega(O)$ et $O$; à l'aide de ces deux fonctions on peut former les deux fonctions $z$ et $z^{\prime}$ par les procédés du $§ 5$. Nous avons vu au $\S 7$ que ces deux fonctions sont liées par une relation linéaire. Done les valeurs de $z$ qui correspondent aux deux points

$$
M_{1}, \Omega\left(M_{1}\right)
$$

sont liées par une relation linéaire. Mais ces deux points correspondent à un 
même point $M \mathrm{du}$ domaine $D$, et par conséquent aux mêmes valeurs des fonctions:

$$
y_{i}=\theta_{i}(z) \quad x=\psi(z) .
$$

Donc ces fonctions $\psi(z)$ et $\theta_{i}(z)$ ne sont pas altérées par certaines relations linéaires. Ces relations linéaires sont généralement en nombre infini, parce que les points de $D^{\prime}$ qui correspondent à un même point de $D$ sont généralement en nombre infini. Elles forment naturellement un groupe; enfin de même que les substitutions des groupes fuchsiens elles n'altèrent pas la circonférence $K$. Ce groupe tout à fait analogue aux groupes fuchsiens peut s'appeler fuchsoïde; ainsi nos fonctions $\psi(z), \theta_{i}(z)$ ne sont pas altérées par les substitutions linéaires d'un groupe fuchsoïde.

\section{$\$$ ro. Relations entre les fonctions de Green.}

Comparons maintenant les fonctions $u_{n}$ relatives au domaine $D$, et celles qui sont relatives au domaine $D^{\prime}$, régulièrement multiple de $D$.

Soit $M$ un point de $D$ et:

$$
\Omega_{1}(M), \Omega_{2}(M), \ldots
$$

les points correspondants de $D^{\prime}$; les fonctions $\Omega_{1}, \Omega_{2}, \ldots$ sont continues, mais il peut se faire qu'elles s'échangent les unes avec les autres quand $M$ décrit une courbe fermée sur $D$.

Considérons la suite des fonctions

$$
u_{0}^{(1)}, u_{1}^{(1)}, \ldots, u_{n}^{(1)}, \ldots
$$

relatives au domaine $D^{\prime}$ et obtenues en partant du point $\Omega_{1}(O)$ et en balayant successivement les éléments

$$
\Omega_{1}\left(C_{1}\right), \Omega_{1}\left(C_{2}\right), \ldots \Omega_{1}\left(C_{n}\right), \ldots
$$

Considérons de même la suite des fonctions

$$
u_{0}^{(k)}, u_{1}^{(k)}, \ldots, u_{n}^{(k)}, \ldots
$$

relatives au domaine $D^{\prime}$ et obtenues en partant de $\Omega_{k}(O)$ et balayant successivement

$$
\Omega_{k}\left(C_{1}\right), \Omega_{k}\left(C_{2}\right), \ldots, \Omega_{k}\left(C_{n}\right), \ldots
$$

D'après le $\S$ précédent on aura, pour un point $M$ quelconque.

$$
u_{n}^{(k)}\left[\Omega_{k}(M)\right]=u_{n}^{\mathrm{i}}\left[\Omega_{1}(M)\right]
$$

Considérons enfin les fonctions:

$$
U_{0}, U_{1}, \ldots, U_{n}, \ldots
$$


Sur l'uniformisation des fonctions analytiques.

relatives au domaine $D$ et obtenues en partant du point $O$ et balayant successivement les éléments suivants de $D$ :

$$
C_{1}, C_{2}, \ldots, C_{n}, \ldots
$$

Je désignerai par $C_{0}$ l'élément de $D$ dont fait partie le point o. Alors le point $\Omega_{k}(O)$ fera partie des éléments $\Omega_{k}\left(C_{0}\right)$. Les différents éléments $\Omega_{k}\left(C_{0}\right)$ n'ont aucun point commun.

Supposons en effet que deux éléments $\Omega_{i}\left(C_{0}\right)$ et $\Omega_{k}\left(C_{0}\right)$ aient un point identique, tous leurs points qui ont mêmes coordonnées devront être identiques, d'après l'une des conditions énoncées au $\$ 2$. Or ces deux éléments coīncident au point de vue géométrique, done tous les points correspondants de ces deux éléments devraient être identiques; si ensuite $\Omega_{i}\left(C_{1}\right)$ est contigu à $\Omega_{i}\left(C_{0}\right), \Omega_{k}\left(C_{1}\right)$ est contigu à $\Omega_{k}\left(C_{0}\right)$; les deux éléments $\Omega_{i}\left(C_{1}\right)$ et $\Omega_{k}\left(C_{1}\right)$ auraient donc des points identiques, et il s'ensuivrait, comme pour $\Omega_{i}\left(C_{0}\right)$ et $\Omega_{k}\left(C_{0}\right)$, que ces deux éléments devraient être identiques; on verrait alors de proche en proche, que quel que soit le point $M, \Omega_{i}(M)$ serait identique à $\Omega_{k}(M)$.

Nous devons donc admettre que les deux éléments $\Omega_{i}\left(C_{0}\right)$ et $\Omega_{k}\left(C_{0}\right)$ ne peuvent avoir aucun point commun, et il en serait de même évidemment pour les deux éléments $\Omega_{i}\left(C_{n}\right)$ et $\Omega_{k}\left(C_{n}\right)$.

Cela posé, je me propose de démontrer que l'on a en un point quelconque du domaine $D^{\prime}$

$$
U_{n}=\sum_{k} u_{n}^{(k)}
$$

et d'abord que

$$
U_{0}=\sum_{k} u_{0}^{(k)}
$$

Considérons un point $M$ quelconque de $D^{\prime}$; s'il n'appartient à aucun des $\Omega_{k}\left(C_{0}\right)$ on aura:

$$
U_{0}=u_{0}^{(k)}=0 \text {. }
$$

S'il appartient à l'un des $\Omega_{k}\left(C_{0}\right)$, il ne pourra appartenir à aucun des $\Omega_{i}\left(C_{0}\right)$, on aura donc:

$$
u_{0}^{(i)}=0 \quad(\text { si } i \gtreqless k)
$$

et d'ailleurs, d'après la façon dont les fonctions $u_{0}$ sont formées:

$$
U_{0}=u_{0}^{(k)}
$$

On aura donc dans tous les cas

$$
U_{0}=\sum_{k} u_{0}^{(k)}
$$

Je dis maintenant que si

$$
U_{n-1}=\sum_{k} u_{n-1}^{(k)}
$$

Acta mathematica. 31. Imprimé le 20 mars 1907. 
on aura également

$$
U_{n}=\sum_{k} u_{n}^{(k)}
$$

En effet si le point $M$ n'appartient à aucun des $\Omega_{k}\left(C_{n}\right)$ c'est à dire à l'un des éléments que l'on balaye pour passer des fonctions d'indice $n-\mathrm{I}$ aux fonctions d'indice $n$, on aura:

$$
U_{n}=U_{n-1} \quad u_{n}^{(k)}=u_{n-1}^{(k)}
$$

Si $M$ appartient à l'un des $\Omega_{k}\left(C_{n}\right)$, il n'appartiendra à aucun autre des $\Omega_{i}\left(C_{n}\right)$; Les masses génératrices de la fonction $U_{n-1}$, s'obtiendront en ajoutant entre elles toutes les masses génératrices des fonctions $u_{n-1}^{(k)}$, en vertu de l'équation (2) et de la définition de ces masses génératrices. En balayant toutes ces masses on aura donc:

$$
U_{n}-U_{n-1}=\sum\left(u_{n}^{(k)}-u_{n-1}^{(k)}\right)
$$

Qu'arrive-t-il maintenant quand $n$ croît indéfiniment; $u_{n}^{(k)}$ tend vers une limite $u^{(k)}$ que nous supposons finie, et $U_{n}$ tend vers une limite $U$ qui peut être infinie. On a donc:

$$
U=U_{0}+\sum_{n}\left(U_{n}-U_{n-1}\right)=\sum_{k} u_{0}^{(k)}+\sum_{n} \sum_{k}\left(u_{n}^{(k)}-u_{n-1}^{(k)}\right)
$$

Il faut ici sommer d'abord par rapport à $k$, puis par rapport à $n$, mais comme les termes de ces séries sont essentiellement positifs, on peut en intervertir l'ordre et écrire:

$$
U=\sum_{k} u_{0}^{(k)}+\sum_{k} \sum_{n}\left(u_{n}^{(k)}-u_{n-1}^{(k)}\right)=\sum_{k} u^{(k)}
$$

Donc la condition nécessaire et suffisante pour que la fonction de GrEwN existe pour le domaine $D$, c'est à dire pour que la suite des $U_{n}$ ait une limite finie c'est que la série $\Sigma_{k} u^{k}$ converge.

$\mathrm{Si}$ cette condition est remplie, nous voyons que les fonctions $U$ et $u^{k}$ étant harmoniques et positives, on aura également, par le théorème de HarNack

et par conséquent:

$$
\frac{d U}{d \xi}=\sum \frac{d u^{(k)}}{d \xi}, \frac{d U}{d \eta}=\sum \frac{d u^{(k)}}{d \eta},
$$

$$
V=\sum v^{(k)} ; Z=\prod z^{(k)}
$$

$v^{(k)}, z^{(k)}, V, Z$ étant formées avec $u^{(k)}, U$, comme $v$ et $z$ l'ont été avec $u$ au $\$ 5$; (la lettre 11 représente un produit infini).

Mais les variables $z^{(k)}$ sont liées entre elles par les relations linéaires, qui forment le groupe fuchsoĩde du $\$$ précédent. On prendra donc les diverses trans- 
formées de z par les substitutions linéaires de ce groupe fuchsoüde, on en fera le produit; si ce produit converge c'est que la fonction de Green existe pour le domaine $D$ et ce produit est précisément la fonction $Z$ déduite de cette fonction de Green $U$ par les procédés $d u \$ 5$.

Soit

\section{§ I I. Propriótés du groupe fuchsoïde.}

$$
\left(z, \frac{\alpha_{i} z+\beta_{i}}{\gamma_{i} z+\delta_{i}}\right)
$$

la substitution la plus générale de notre groupe fuchsoïde. Désignons par $u(D, O)$ la fonotion de GreEN relative au domaine $D$ et qui devient infinie au point $O$. De cette façon; les fonctions

$$
u^{(k)}, U
$$

du § précédent seront désignées par

$$
u\left[D^{\prime}, \Omega_{k}(O)\right], u(D, O) .
$$

Nous désignerons par $z(D, O)$ la fonction déduite de $u(D, O)$ comme $z$ a été déduit de $u$ au paragraphe 5 .

Nous désignerons simplement par $z$ la fonction $z\left[D^{\prime}, \Omega_{1}(0)\right]$ et ce sera notre nouvelle variable indépendante. Nous aurons alors:

$$
z\left[D^{\prime}, \Omega_{k}(O)\right]=\frac{\alpha_{k} z+\beta_{k}}{\gamma_{k} z+\delta_{k}} .
$$

le second membre correspondant à l'une des substitutions du groupe fuchsoïde; d'après cela, on devra avoir $\alpha_{1}=\delta_{1}=1, \beta_{1}=\gamma_{1}=0$, de sorte que l'indice I correspondra à la substitution identique.

On aura alors, d'après le $\S$ précédent:

$$
z(D, O)=\prod \frac{\alpha_{k} z+\beta_{k}}{\gamma_{k} z+\delta_{k}}
$$

Soit maintenant $\Omega_{1}\left(O^{\prime}\right)$ un autre point quelconque de $D^{\prime}$, on aura d'après le $\$ 7$

$$
z\left[D^{\prime}, \Omega_{1}\left(O^{\prime}\right)\right]=\frac{a z+b}{c z+d}
$$

$a, b, c, d$ étant des constantes; on en conclut

$$
z\left[D^{\prime}, \Omega_{k}\left(O^{\prime}\right)\right]=\frac{a\left(\alpha_{k} z+\beta_{k}\right)+b\left(\gamma_{k} z+\delta_{k}\right)}{c\left(\alpha_{k} z+\beta_{k}\right)+d\left(\gamma_{k} z+\delta_{k}\right)}
$$

et on trouvera encore: 
(2)

$$
z\left(D, O^{\prime}\right)=\prod z\left[D^{\prime}, \Omega_{k}\left(O^{\prime}\right)\right]=\prod \frac{a\left(\alpha_{k} z+\beta_{k}\right)+b\left(\gamma_{k} z+\delta_{k}\right)}{c\left(\alpha_{k} z+\beta_{k}\right)+d\left(\gamma_{k} z+\delta_{k}\right)} .
$$

Il s'agit done de savoir si la somme

$$
u(D, O)=\boldsymbol{\Sigma} \log \left|\frac{x_{k} z+\beta_{k}}{\gamma_{k} z+\delta_{k}}\right|
$$

converge, c'est à dire si la fonction de GrEEN existe pour le domaine $D$.

Or en vertu d'un théorème bien connu la condition nécessaire et suffisante pour que cette série

$$
\Sigma \log \left|\frac{\alpha z+\beta}{\gamma z+\delta}\right|
$$

converge, c'est que la série

$$
\boldsymbol{\Sigma}\left(1-\left|\frac{\alpha z+\beta}{\gamma z+\delta}\right|\right)
$$

converge absolument, ou encore que la série:

$$
\mathbf{\Sigma}\left(1--\left|\frac{\alpha z+\beta}{\gamma z+\delta}\right|^{2}\right)
$$

converge ou encore qu'il en soit ainsi de la série:

$$
\mathbf{\Sigma}\left[r-\frac{(\alpha z+\beta)\left(\gamma z^{\prime}+\delta\right)}{(\gamma z+\delta)\left(\alpha z^{\prime}+\beta\right)}\right]
$$

en désignant par $z^{\prime}$ une quantité qui a même argument que $z$ et même module que $\frac{\mathrm{I}}{z}$; et en effet

$$
\frac{\alpha z+\beta}{\gamma z+\delta}, \frac{\gamma z^{\prime}+\delta}{\alpha z^{\prime}+\beta}
$$

sont imaginaires conjuguées. Or cela peut s'écrire (en tenant compte de $\alpha \delta-\beta \gamma=1$ );

$$
\mathbf{\Sigma} \frac{z-z^{\prime}}{(\gamma z+\delta)\left(\alpha z^{\prime}+\beta\right)} .
$$

Il est aisé de voir que pour les termes d'ordre élevé, pour lesquels $\alpha, \beta, \gamma, \delta$ ont de très grandes valeurs, $\left|\alpha z^{\prime}+\beta\right|$ est sensiblement égal à $\left|\gamma z^{\prime}+\delta\right|$ et à $|\gamma z+\delta|$.

Ainsi la condition nécessaire et suffisante pour que la fonction de Green existe c'est que la série:

$$
\sum \frac{1}{|\gamma z+\delta|^{2}}
$$

converge. 
Reportons-nous au premier chapitre de mon mémoire sur les fonctions fuchsiennes et rappelons un des modes de raisonnement que nous y avons employés; soit $S$ une petite aire enveloppant le point $z$, et soit $S_{k}$ (loco citato) ce que devient cette aire quand on change $z$ en $\frac{\alpha_{k} z+\beta_{k}}{\gamma_{k} z+\delta_{k}}$. Nous avons vu que ces aires sont entre elles comme les quantités

$$
\frac{\mathrm{I}}{\left|\gamma_{k} z+\delta_{k}\right|^{4}}
$$

et comme la série $\sum S_{k}$ converge certainement, puisque la somme de toutes ces aires est toujours plus petite que le cercle fondamental, nous en avons conclu que la série

$$
\sum \frac{1}{|\gamma z+\delta|^{4}}
$$

converge toujours, ce qui est le fondement de la théorie des fonctions thétafuchsiennes.

Dans le cas qui nous occupe, nous pouvons en tirer la conclusion suivante: La condition nécessaire et suffisante pour que la fonction de Green existe pour le domaine $D$, c'est que la série

converge.

$$
\sum \sqrt{S_{k}}
$$

Si cette condition est remplie, et si $D$ est simplement connexe, il existe une fonction $Z$ qui prend aux différents points du domaine $D$ toutes les valeurs intérieures au cercle de rayon I et ne prend chacune d'elles qu'une fois. Elle permet la représentation conforme du domaine $D$ sur un cercle. Elle n'est pas alterée par les substitutions du groupe fuchsoïde.

Si cette condition est remplie, et si $D$ n'est pas simplement connexe, il existe une fonction $Z$ qui par rapport aux substitutions du groupe fuchsoïde satisfait à la condition:

$$
Z\left(\frac{\alpha_{k} z+\beta_{k}}{\gamma_{k} z+\delta_{k}}\right)=M_{k} Z(z)
$$

$M_{k}$ étant une constante de module I.

Qu'arrive-t-il enfin si la condition n'est pas remplie. On pourrait former les séries thétafuchsiennes, lambdafuchsiennes, etc., tout comme avec les fonctions fuchsiennes ordinaires; on arriverait ainsi à l'étude complète du domaine $D$. Maís nous suivrons une autre voie. 


\section{§ 12. Le domaine $D_{1}$ et sa fonetion de Green.}

Je vais utiliser les résultats de la méthode alternante de M. Schwarz. Reprenons notre domaine $D$, sur lequel nous ne faisons aucune hypothèse. Soit $C_{0}$ un élément de ce domaine, où se trouvera le point $O$, et $C_{0}^{\prime}$ un autre élément qui aura pour centre le point $O^{\prime}$. Nous pourrons toujours supposer que le point $O$ qui appartient à $C_{0}$, n'appartient à aucun autre élément de $D$, contigu à $C_{0}$, et de même que le point $O^{\prime}$ qui appartient à $C_{0}^{\prime}$ n'appartient à aucun autre élément de $D$, contigu à $\mathrm{C}_{0}^{\prime}$.

Construisons ensuite un domaine $D_{1}$ contenu dans $D$, et qu'on déduira de $D$ tout simplement en supprimant l'élément $C_{0}^{\prime}$; les points de $D$ qui font partie de $C_{0}^{\prime}$, sans appartenir à aucun autre élément de $D$, contigu à $C_{0}^{\prime}$ ne feront pas partie de $D_{1}$; et au contraire les points des éléments contigus à $C_{0}^{\prime}$ appartiendront à $D_{1}$, bien que faisant partie de $C_{0}^{\prime}$.

Je dis d'abord que la fonction de GreEN existera pour ce domaine $D_{1}$. Formons en effet comme au $\S 3$, la série des fonctions $u_{n}$ en partant du point $O$ et en effectuant une série de balayages successifs portant sur les divers éléments de $D_{1}$ (c'est à dire sur les divers éléments de $D$, sauf sur $C_{0}^{\prime}$ ). Ces fonctions $u_{n}$ sont des potentiels logarithmiques engendrés par une masse positive $+I$ située en $O$ et par diverses masses négatives dont la somme est égale à - $-\mathrm{I}$.

Cherchons la valeur moyenne de $u_{n}$ sur la circonférence de $C_{0}^{\prime}$; prenons pour un instant des coordonnées polaires $r$ et $\omega$, en prenant pour pôle le centre de $C_{0}^{\prime}$, c'est à dire le point $O^{\prime}$, et envisageons l'intégrale:

$$
J=\int u_{n} d \omega
$$

prise le long d'une circonférence ayant son centre en $O^{\prime}$; nous aurons:

$$
r \frac{d J}{d r}=\int \frac{d u_{n}}{d r} r d \omega .
$$

Cette intégrale est, en vertu des théorèmes de Gresn, égale à $-2 \pi$ multiplié par la somme des masses génératrices situées à l'intérieur de la circonférence. Cette somme est plus petite que I en valeur absolue; on a done:

$$
2 \pi>r \frac{d J}{d r}>0
$$

Si alors $r_{0}$ est le rayon de la circonférence de $C_{0}$ et si $r_{1}$ est la plus courte distance de $O^{\prime}$ à la frontière de $D_{1}$, on aura: 


$$
J_{0}<J_{1}+2 \pi \log \frac{r_{0}}{r_{1}} .
$$

Mais $J_{1}$ est nul puisque $u_{n}$ est nul en tous les points de $C_{0}^{\prime}$ qui ne font pas partie de $D_{1} ; r_{1}$ n'est pas nul, car le point $O^{\prime}$ et les points voisins ont été supposés en dehors de $D_{1}$; on a donc:

$$
J_{0}=\int u_{n} d \omega<2 \pi \log \frac{r_{0}}{r_{1}}
$$

ce qui veut dire que la valeur moyenne de $u_{n}$ sur la circonférence de $C_{0}^{\prime}$ est plus petite que $\log \frac{r_{0}}{r_{1}}$; ̀̀ fortiori la valeur minimum de $u_{n}$ sur cette circonférence sera plus petite que $\log \frac{r_{0}}{r_{1}}$.

Soit $E_{n}$ l'ensemble des arcs de cette circonférence tels que

$$
u_{n}<\log \frac{r_{0}}{r_{1}}
$$

On voit que $E_{n}$ ne peut pas se réduire à rien, quel que soit $n$. De plus $E_{n+1}$ est contenu dans $E_{n}$ puisque

$$
u_{n+1}>u_{n}
$$

Donc il y a sur la circonférence de $C_{0}^{\prime}$ au moins un point $M$ tel que l'on ait, pour toutes les valeurs de $n$

$$
u_{n}<\log \frac{r_{0}}{r_{1}}
$$

En ce point $M$ la suite des $u_{n}$ convergera; et comme elle ne peut converger en un point sans converger partout (voir $\$ 3$ ) elle convergera dans tout le domaine $D_{1}$. La fonction de Green que nous appellerons $u\left(D_{1}, O\right)$ existe donc pour ce domaine $D_{1}$.

Soit $\delta$ la partie commune à $C_{0}^{\prime}$ et à $D_{1}$; ce sera un domaine limité extérieurement par la circonférence $C_{0}^{\prime}$ et intérieurement par une ligne fermée $L_{0}$, formée d'un nombre fini d'ares de cercle appartenant aux circonférences des divers éléments contigus à $C_{0}^{\prime}$. Cette ligne fermée $L_{0}$ fera partie de la frontière de $D_{1}$.

On peut vérifier que la fonction de GrEEN $u\left(D_{1}, O\right)$ tend vers zéro quand on se rapproche de $L_{0}$. En effet, cette fonction est harmonique et par conséquent analytique sur la circonférence $C_{0}^{\prime}$ qui est tout entière à l'intérieur de $D_{1}$. Nous pouvons done construire une fonction $T$ qui soit harmonique dans le domaine $\delta$, qui soit égale à $O$ sur $L_{0}$ et à $u\left(D_{1}, O\right)$ sur la circonférence $C_{0}$. On aura alors 
sur $L_{0}$ et

$$
u_{n}=T=0
$$

$$
u_{n}<T
$$

sur la circonférence $C_{0}^{\prime}$. Comme $u_{n}-T$ ne peut admettre que des minima, on aura:

$$
u_{n}<T
$$

dans tout le domaine $\delta$; et par conséquent

$$
0<u\left(D_{1}, O\right) \leq T .
$$

Mais quand on se rapproche de $L_{0}, T$ tend vers zéro, il en est donc de même de $u\left(D_{1}, O\right)$. Donc $T$ et $u\left(D_{1}, O\right)$ sont deux fonctions harmoniques dans le domaine $\delta$ et qui prennent les mêmes valeurs sur la frontière de ce domaine. Elles sont donc égales et l'on a:

$$
u\left(D_{1}, O\right)=T .
$$

Cela nous prouve en même temps, qu'en tous les points de $L_{0}$, la dérivée $\frac{d u\left(D_{1}, O\right)}{d n}$ se comporte régulièrement, puisqu'il en est ainsi de $\frac{d T}{d n}$; et il en est ainsi de $\frac{d T}{d n}$, puisque le domaine dans lequel la fonction $T$ est définie est limité par un nombre fini d'arcs de cercle et que tous les raisonnements habituels relatifs au problème de DiRICHLET lui sont applicables sans difficulté.

Cela posé, supposons que le domaine $D$ soit simplement connexe, le domaine $D_{1}$ ne le sera pas en général, car par exemple la circonférence $C_{0}^{\prime}$ limite une aire faisant partie de $D$, à savoir le cercle $C_{0}^{0}$, mais cette aire ne fait pas partie de $D_{1}$. Pour rendre le domaine $D_{1}$ simplement connexe, nous allons y pratiquer une coupure $Q$. La coupure $Q$ sera une ligne qui partira d'un point quelconque de $L_{0}$ et qu'on prolongera indéfiniment dans le domaine $D_{1}$.

Je précise; imaginons une série de domaines successifs que j'appellerai

$$
d_{1}, d_{2}, d_{3}, \ldots
$$

définis de la façon suivante; chacun d'eux sera formé d'un nombre fini d'éléments de $D_{1} ; d_{1}$ comprendra entre autres tous ceux de ces éléments qui sont contigus à $C_{0}^{0} ; d_{n+1}$ comprendra tous les éléments de $d_{n}$ et d'autres encore; enfin nous nous arrangerons pour que le domaine comprenant $C_{0}^{\prime}$ et tous les éléments de $d_{n}$ et que j'appellerai $e_{n}$ soit simplement connexe, et que tout élément de $D_{1}$ fasse partie de l'un des domaines $d_{n}$ (et par conséquent de tous les domaines suivants $\left.d_{n+1}, \ldots\right)$

Le domaine $e_{n}$ étant simplement connexe sera limité par une certaine courbe fermée $L_{n}$; alors le domaine $d_{n}$ sera doublement connexe et compris entre les deux 
courbes $L_{n}$ et $L_{0}$; le domaine $d_{n+1}-d_{n}$ sera doublement connexe et compris entre les deux courbes $L_{n+1}$ et $L_{n}$.

Alors notre coupure $Q$ sera composée d'une série d'ares $Q_{1}, Q_{2}, \ldots, Q_{n}$, etc., l'arc $Q_{n}$ allant de $L_{n-1}$ à $L_{n}$ à travers le domaine $d_{n}-d_{n-1}$. Dans ces conditions la coupure $Q$ rend le domaine $D_{1}$ simplement connexe, et en effet si une courbe fermée quelconque tracée sur $D_{1}$, n'enferme pas une aire appartenant à $D_{1}$, cette courbe appartiendra à l'un des domaines $d_{n}$ et enveloppera la ligne $L_{0}$, elle coupera donc la coupure $Q_{1}+Q_{2}+\cdots+Q_{n}$, et par conséquent la coupure $Q$.

Soit $D_{2}$ le domaine obtenu en partant de $D_{1}$ et le rendant simplement connexe par l'introduction de la coupure $Q$. Il va sans dire que certains éléments de $D_{1}$ étant traversés par la coupure $Q$, devront être remplacés dans le domaine $D_{2}$ par d'autres éléments en nombre fini ou infini, disposés de telle sorte qu'aucun de ces éléments nouveaux ne soit traversé par $Q$ et que tous les points de l'élément ancien, sauf ceux de la coupure $Q$, appartiennent au moins à l'un des éléments nouveaux.

Cela posé je dis que la fonction de Green $u\left(D_{2}, O\right)$ existe pour le domaine $D_{2}$; en effet soit

$$
u_{n}\left(D_{2}, O\right)
$$

une des fonctions obtenues par les balayages successifs, on aura:

$$
u_{n}\left(D_{2}, O\right)<u\left(D_{1}, O\right)
$$

puisque la dernière fonction est harmonique et positive dans tout le domaine $D_{1}$ et par conséquent dans tout le domaine $D_{2}$; que $u_{n}$, (à part la masse + I qui est au point $O$ ) n'est engendré que par des masses négatives de telle façon que la différenee

$$
u_{n}\left(D_{2}, O\right)-u\left(D_{1}: O\right)
$$

ne peut admettre que des minima et pas de maxima et est d'ailleurs négative dans tous les éléments où $u_{n}\left(D_{2}, 0\right)$ est nul, c'est à dire dans tous les éléments de $D_{2}$ sauf un nombre fini d'entre eux.

Donc la suite des $u_{n}\left(D_{2}, O\right)$ converge.

C. Q. F. D.

Le domaine $D_{2}$ étant simplement connexe, l'existence de la fonction $u\left(D_{2}, O\right)$ entraîne celle de la fonction $z\left(D_{2}, O\right)$, c'est à dire d'après les $\S 5,6,7,8$ la possibilité de la représentation conforme du domaine $D_{2}$ sur un cercle $K$. On voit alors, que la circonférence de $K$ va se partager en quatre arcs; l'un l'arc $A B$ correspondra ̀̀ la ligne $L_{0}$, les deux extrémités $A$ et $B$ correspondant au point où la coupure $Q$ aboutit à $L_{0}$; deux autres arcs $A^{\prime} A$ et $B B^{\prime}$ correspondront aux deux lèvres de la coupure $Q$. Le quatrième arc $B^{\prime} A^{\prime}$ correspondrait à la Acta mathematica. 31. Imprimé le 21 mars 1907. 
frontière du domaine primitivement donné $D$. Mais ce dernier arc $B^{\prime} A^{\prime}$ peut se réduire à un point.

Nous sommes donc amenés à distinguer deux cas:

I $^{\circ}$. Celui où l'arc $B^{\prime} A^{\prime}$ ne se réduit pas à un point. Dans ce cas je dis que le domaine $D_{1}$ est susceptible d'une représentation conforme sur un anneau circulaire et le domaine $D$ sur un cercle; la fonction de Grees $u(D, O)$ existe.

$2^{\circ}$. Celui où l'arc $B^{\prime} A^{\prime}$ se réduit à un point. Dans ce cas je dis que le domaine $D_{1}$ est susceptible de représentation conforme sur un cercle, et le domaine $D$ sur une sphère fermée.

\section{§ 13. La fonetion $\boldsymbol{V}$.}

Proposons-nous d'abord de construire une fonction $V$ qui soit harmonique dans le domaine $D_{1}$, égale à I sur $L_{0}$ et qui tende vers zéro quand on se rapproche de la frontière du domaine primitivement donné $D$. Partons d'une fonction $V_{0}$ ainsi définie:

$\mathrm{I}^{\circ}$. A l'intérieur de $L_{0}$, (c'est à dire dans la partie de $D$ qui ne fait pas partie de $D_{1}$ ) on a $V_{0}=\mathrm{I}$.

$2^{0}$. Sur $L_{0}$ on a $V_{0}=\mathrm{I}$.

$3^{0}$. En dehors de $C_{0}^{\prime}$ et sur la circonférence de $C_{0}^{\prime}$ on a $V_{0}=0$.

$4^{0}$. Dans le domaine $\delta$, c'est à dire entre la circonférence $C_{0}^{\prime}$ et $L_{0}$, la fonction $V_{0}$ est harmonique, elle tend vers zéro quand on se rapproche de la circonférence $C_{0}^{\prime}$ et vers I quand on se rapproche de $L_{0}$; elle est donc toujours comprise entre o et $\mathrm{I}$.

Nous allons maintenant en partant de cette fonction $V_{0}$, balayer successivement les divers éléments de $D_{1}$.

Cette fonction $V_{0}$ se comporte comme un potentiel logarithmique engendré par diverses masses situées sur $L_{0}$ e à l'intérieur de $L_{0}$ (donc en dehors des éléments de $D_{1}$ ) et sur lesquelles par conséquent ne porteront pas les balayages, et par des masses négatives situées sur la circonférence de $C_{0}^{\prime}$.

Les masses balayées étant toutes négatives, les fonctions $V_{n}$ seront toujours croissantes, toujours comprises entre $O$ et $I$. La suite des $V_{n}$ convergera donc vers une certaine limite $V$ qui sera harmonique dans le domaine $D_{1}$.

$\mathrm{Je}$ dis que $V$ tend vers I quand on se rapproche de $L_{0}$. Soit en effet $\lambda$ un des $\operatorname{arcs}$ de $L_{0}$, soit $c$ la circonférence à laquelle cet arc appartient et qui limitera un cercle $c$ correspondant à un élément de $D$ contigu à $C_{0}^{\prime}$; je suppose d'abord que l'on se rapproche de l'arc $\lambda$, je construis une fonction $T$, harmonique 
à l'intérieur de $c$, égale à $\mathrm{I}$ sur $\lambda$ et à $\mathrm{O}$ sur le reste de la circonférence $c$. Au moment où on vient de balayer $c$, la fonction $V_{n}$ est harmonique dans c; et on a:

$$
\begin{array}{ll}
V_{n}=T=\mathrm{I} & \text { sur } \lambda \\
V_{n}>0=T & \text { sur le reste de la circonférence } c .
\end{array}
$$

On a donc à l'intérieur de $c$

$$
\mathrm{I}>V_{n}>T
$$

et par conséquent à la limite:

$$
\mathrm{I}>V>T \text {. }
$$

Quand on se rapproche de $\lambda, T$ tend vers $I$, il en est donc de même de $V$. Il reste à voir ce qui se passe quand on se rapproche de l'extrémité d'un des $\operatorname{arcs} \lambda$, c'est à dire du point d'intersection de deux circonférences telles que $c$. Il n'est pas nécessaire pour cela de répéter la discussion. Soient $c$ et $c^{\prime}$ ces deux circonférences, $A$ leur point d'intersection, $\lambda$ et $\lambda^{\prime}$ les deux arcs de $L_{0}$ qui font partie de $c$ et $c^{\prime}$; du point $A$ comme centre décrivons un cercle $\gamma$ de rayon très petit, et considérons le domaine $\varepsilon$ commun à $\gamma$ et à $D_{1}$ et qui sera limité par une partie de la circonférence de $\gamma$ et par une partie des $\operatorname{arcs} \lambda$ et $\lambda^{\prime}$; dans ce domaine la fonction $V$ est harmonique, elle tend vers I quand on se rapproche d'un quelconque des points de $\lambda$ ou de $\lambda$ ', le point $A$ excepté; cela suffit, d'après les propriétés bien connues des fonctions harmoniques, pour qu'elle tende encore vers I quand on se rapproche du point $A$.

On pourrait seulement se demander si la fonction $V$ ne se réduit pas à une constante et n'est pas partout égale à $\mathrm{I}$.

Supposons d'abord qu'il n'en soit pas ainsi. Je dis qu'en tout point intérieur au domaine $D_{1}$ elle est plus petite que I. Considérons en effet la représentation conforme du domaine $D_{2}$ défini plus haut sur le cercle $K$. Nous voyons que la fonction $V$ (considérée non plus comme fonction d'un point $N$ du domaine $D_{1}$ (ou $D_{2}$ ) mais comme fonction du point correspondant $M$ du cercle $K$ ) est encore harmonique; elle est d'ailleurs toujours comprise entre o et $\mathrm{r}$. De plus en un point $M$ au moins intérieur à $K$, la fonction $V$ est $<\mathrm{I}$, puisque par hypothèse elle ne se réduit pas identiquement à $\mathrm{I}$. Soit alors $M^{\prime}$ un autre point intérieur à $K$; soient $V(M)$ et $V\left(M^{\prime}\right)$ les valeurs de $V$ en ces deux points. Comme la fonction harmonique I $-V$ est toujours positive, nous pouvons appliquer le théorème de HARNACK et assigner une limite supérieure au rapport

$$
\frac{\mathrm{I}-V\left(M^{\prime}\right)}{\mathrm{I}-V(M)} \text {. }
$$

Comme I $-V(M)$ n'est pas nul, il en est de même de $-V\left(M^{\prime}\right)$. Ainsi la fonction $V$ n'est pas égale à $\mathrm{I}$, sauf peut être aux points de $D_{1}$ (ou de $D_{2}$ ) qui 
correspondent à un point situé sur la circonférence de $K$. Mais les seuls points intérieurs à $D_{1}$ qui correspondent à un point de la circonférence de $K$ sont ceux qui appartiennent à la frontière de $D_{2}$, c'est à dire les points de la coupure $Q$. Mais comme cette coupure $Q$ a été arbitrairement tracée, et qu'on peut la changer, le théorème est vrai pour tous les points intérieures à $D_{1}$.

En particulier on peut assigner une limite inférieure différente de zéro ì la différence $\mathrm{I}-V$ sur la circonférence de $C_{0}^{\prime}$.

Nous aurions pu au lieu d'appliquer les procédés précédents au domaine $D_{1}$, les appliquer an domaine $d_{n}$ que nous avons défini plus haut et qui est limité par les lignes $L_{n}$ et $L_{0}$ (Cf. page 49) Nous aurions obtenu une fonction $V\left(d_{n}\right)$ qui aurait été à $d_{n}$ ce que $V$ est à $D_{1}$.

Ces fonctions $V\left(d_{n}\right)$ présentent une grande analogie avec les fonctions $u_{p 0}$ du $\S 6$; on pourrait les obtenir en effet en partant de $V$ et en balayant une infinité de fois tous les éléments de $d_{n}$ avant de passer au balayage des autres éléments de $D_{1}$.

L'essentiel c'est que

$$
V\left(d_{n}\right)
$$

va en croissant avec $n$; l'application du théorème de HaRnack nous apprend alors que la suite des $V\left(d_{n}\right)$ converge uniformément vers $V$, et que, [si $V^{\prime}$ est une dérivée quelconque de $V$ et $V^{\prime}\left(d_{n}\right)$ la dérivée correspondante de $V\left(d_{n}\right)$ ], la suite des $V^{\prime}\left(d_{n}\right)$ converge uniformément vers $V^{\prime}$.

Posons maintenant:

$$
K_{n}=-\int \frac{d V\left(d_{n}\right)}{d r} d s, \quad K=-\int \frac{d V}{d r} d s
$$

Les intégrales sont prises le long de la circonférence de $C_{0}^{\prime} ; d s$ est l'élément d'arc de cette circonférence; les dérivées $\frac{d V}{d r}, \frac{d V\left(d_{n}\right)}{d r}$ sont prises par rapport à la normale à cette circonférence; il résulte de ce qui précède que la suite des $K_{n}$ converge vers $K$; nous poserons alors:

$$
W=\frac{2 \pi V}{K} \quad W_{n}=\frac{2 \pi V\left(d_{n}\right)}{K_{n}} .
$$

On voit que la suite des $W_{n}$ converge uniformément vers $W$, et que la suite des $W_{n}^{\prime}$ converge uniformément vers $W^{\prime}$, (en désignant par $W$ et $W_{n}^{\prime}$ une dérivée quelconque de $W$ et de $W_{n}$ ).

Nous allons maintenant définir des fonctions qui seront à $W$ et $W_{n}$ ce que $v$ et $z$ étaient à $u$ au $\$ 5$. Posons à cet effet, comme dans ce $\$ 5$ : 


$$
\begin{aligned}
& \frac{d W^{\prime}}{d \xi}=-\frac{d W}{d \eta}, \quad \frac{d W}{d \xi}=\frac{d W^{\prime}}{d \eta} \\
& \frac{d W_{n}^{\prime}}{d \xi}=-\frac{d W_{n}}{d \eta}, \quad \frac{d W_{n}}{d \xi}=\frac{d W_{n}^{\prime}}{d \eta} \\
& Z=e^{-\left(W+i W^{\prime}\right)}, \quad Z_{n}=e^{-\left(W_{n}+i W_{n}^{\prime}\right)} .
\end{aligned}
$$

Nous voyons que $Z_{n}$ et ses dérivées convergent uniformément vers $Z$ et ses dérivées. Les fonctions $Z$ et $Z_{n}$ sont uniformes, en effet, quand on tourne autour de $L_{0}, W$ et $W_{n}^{\prime}$ augmentent de $2 \pi$, tandis que $Z$ et $Z_{n}$ ne changent pas.

La variable $Z_{n}$ nous donne la répresentation conforme du domaine $d_{n}$ sur un anneau circulaire; en effet la frontière de $d_{n}$ se compose de $L_{0}$ et de $L_{n}$, sur $L_{0}$ on a $V_{n}=\mathrm{I}, W_{n}=\frac{2 \pi}{K_{n}}$, et sur $L_{n}$ on a $W_{n}=0$; il n'y a pas de difficulté pour établir ce dernier point puisque les lignes $L_{0}$ et $L_{n}$ qui limitent le domaine $d_{n}$ se composent d'un nombre fini d'arcs de cercle. Il résulte de là que $Z_{n}$ ne peut prendre plus d'une fois la valeur $A$ et par conséquent que l'intégrale

$$
J_{n}=\int \frac{d Z_{n}}{Z_{n}-\bar{A}}
$$

prise le long d'un contour fermé quelconque ne peut être égale qu'à zéro ou à $2 i \pi$. Mais nous avons vu que $Z_{n}$ et ses dérivées convergent uniformément vers $Z$ et ses dérivées, d'où il suit que $J_{n}$ tend vers

$$
J=\int \frac{d Z}{Z-A},
$$

ce qui montre que cette intégrale ne peut être égale qu'à zéro ou $2 i \pi$, et que $Z$ ne peut pendre plus d'une fois la valeur $A$.

Donc la variable $Z$ nous donne la représentation conforme du domaine $D_{1}$ sur un domaine plan, à un seul feuillet, c'est à dire dont les diverses parties ne se recouvrent pas mutuellement et qui est limité intérieurement par la circonférence de rayon $e^{-\frac{2 \pi}{\bar{K}}}$ et extérieurement par une ligne fermée ne pouvant sortir de la circonférence de rayon I (ce dernier point provient de ce que $W$ est essentiellement positif).

Il faut maintenant établir que cette ligne fermée, qui limite extérieurement ce domaine plan n'est autre chose que la circonférence de rayon I. C'est un problème analogue à celui du $\S 8$. Nous n'avons qu'à répéter l'analyse de ce $\$$ que nous ne reproduirons pas. L'ensemble $E$ des points du plan auxquels correspond un point de $D_{1}$ n'est plus simplement connexe, puisque $D_{1}$ n'est pas 
simplement connexe; mais il est doublement connexe, étant limité intérieurement par la circonférence de rayon $e^{-\frac{2 \pi}{\bar{K}}}$; cette circonférence et une courbe fermée tracée dans $E$ limitent alors toujours une aire faisant partie de $E$; si done une circonférence de même centre, a tous ses points dans $E$, il ne pourra y avoir à l'intérieur de cette circonférence aucun point de l'ensemble $E_{1}$ des points du plan extérieurs à la circonférence ce rayon $e^{-\frac{2 \pi}{K}}$ et n'appartenant pas à $E$.

En s'appuyant là dessus on définira comme au $\$$ la fonction auxiliaire de OSGOOD $\varphi(w)$.

Nous prendrons ensuite

$$
t=A \log \left|\frac{\mathrm{I}}{w}\right|
$$

le coëfficient $A$ étant assez grand pour que $t$ soit plus grand que $\frac{2 \pi}{\bar{K}}$ c'est à dire que $W_{n}$ le long de la circonférence de rayon $e^{-\frac{2 \pi}{K}}$ qui limite interieurement $E$. Le reste de la démonstration se poursuit comme au $\$ 8$ et on arrive à la même conclusion: tout point $q$ de la frontière de $E$ est sur la circonférence de rayon I.

Done la fonction $Z$ nous donne la représentation conforme de $D_{1}$ sur un anneau circulaire.

Nous avons ainsi:

$1^{\circ}$. Le domaine $D_{1}$ applicable sur un anneau circulaire.

$2^{\circ}$. L'élément $C_{0}^{\prime}$ qui est un cercle.

Ces deux domaines ont une partie commune $\delta$ et leur ensemble forme le domaine

$$
D=D_{1}+C_{0}^{\prime}-\delta .
$$

On peut alors appliquer un théorème connu de M. Schwarz et conclure que $D$ est applicable sur un cercle. La fonction de GREEN $u(D, O)$ existe.

Où dans le raisonnement qui précéde, avons-nous été amenés à supposer que la fonction $V$ ne se réduit pas à une constante égale à I; c'est que si $V$ se réduisait à $\mathrm{I}$, la quantité $K$ serait nulle. Ce qui rendrait la démonstration illusoire. Supposons donc que $V$ ne se réduit pas à $\mathrm{I}$, je dis que $K$ ne sera pas nul. Nous avons en effet comme nous l'avons vu plus haut, une limite supérieure de $V$, plus petite que 1 sur la circonférence $C_{0}^{\prime}$, c'est ce que nous avons expliqué plus haut, soit $A$ cette limite; soit alors $T$ une fonction harmonique dans le domaine $\bar{\delta}$, se réduisant à 1 sur $L_{0}$ et à $A$ sur la circonférence $C_{0}^{\prime}$; on aura

$$
K>-\int \frac{d T}{d r} d s
$$

et l'intégrale $d u 2^{d}$ membre n'est pas nulle. 


\section{$\S$ I 4. Seconde démonstration.}

On peut arriver au même résultat d'une façon plus simple par une autre voie. Observons d'abord que nous pouvons supposer que la ligne $L_{0}$, qui limite $D_{1}$ se réduit à une circonférence concentrique à $C_{0}^{\prime}$. En effet rien ne nous oblige à prendre pour limite de $D_{1}$, les frontières de divers éléments de $D$, puisque ces éléments ont été tracés sur $D$ d'une façon très arbitraire et qu'on aurait pu remplacer $D$ par un domaine équivalent au sens du $\$ 2$. Si nous avions supposé plus haut que $L_{0}$ est une circonférence, plusieurs des raisonnements précédents auraient pu être simplifiés.

Soit $O^{\prime}$ le centre commun de $C_{0}^{\prime}$ et de $L_{0}, r_{0}$ le rayon de $L_{0}, r_{1}$ celui de $C_{0}^{\prime}, r$ la distance d'un point quelconque à $o^{\prime}$. Considérons la fonction $V$, elle est égale à I sur $L_{0}$, et $\operatorname{sur} C_{0}^{\prime}$ elle est $<A<\mathrm{I}$; je dis que nous pouvons assigner une limite inférieure à la dérivée

$$
-\frac{d V}{d r}
$$

en un point quelconque de $L_{0}$; cette expression est d'ailleurs essentiellement positive; et en effet cette limite inférieure est

Je l'appellerai $q$.

$$
\frac{\mathrm{I}-A}{r_{0} \log \frac{r_{1}}{r_{0}}}
$$

Soit ensuite à l'intérieur de $L_{0}$ :

$$
T=\log \frac{r_{0}}{r} .
$$

Nous aurons sur $L_{0}$

$$
T=\circ, \quad-\frac{d T}{d r}=\frac{\mathrm{I}}{r_{0}} .
$$

Construisons alors une fonction $U$ satisfaisant aux conditions suivantes:

${ }^{\circ}$. Dans $D_{1}$ on a

$$
U=\frac{V}{q r_{0}}
$$

$2^{\circ}$. A l'intérieur de $L_{0}$ on a:

$$
U=T+\frac{\mathrm{I}}{q r_{0}} .
$$

$3^{\circ}$. Sur $L_{0}$ on a

$$
U=\frac{\mathrm{I}}{q r_{0}}=\frac{V}{q r_{0}}=T+\frac{\mathrm{I}}{q r_{0}} .
$$


Considérons deux points très voisins de $L_{0}$, l'un à l'extérieur, l'autre à l'intérieur; au $\mathrm{r}^{\text {er }}$ on aura:

$$
-\left(\frac{d U}{d r}\right)_{0}=\frac{-1}{q r_{0}} \frac{d V}{d r}>\frac{1}{r_{0}}
$$

au second on aura

$$
-\left(\frac{d U}{d r}\right)_{1}=-\frac{d T}{d r}=\frac{1}{r_{0}}
$$

et par conséquent

$$
-\left(\frac{d U}{d r}\right)_{0}>-\left(\frac{d U}{d r}\right)_{1}
$$

ce qui veut dire que la fonction $U$, harmonique dans $D_{1}$ et à l'intérieur de $L_{v}$, présente sur $L_{0}$ une discontinuité correspondant à des "masses génératrices» positives.

Cela posé, je dis que la fonction de Green

$$
u\left(D, O^{\prime}\right)
$$

existe; formons en effet par balayages successifs les fonctions

$$
u_{n}\left(D, O^{\prime}\right)
$$

elles admettent une masse $+I$ en $O^{\prime}$, et en outre des masses génératrices qui sont toutes négatives. La différence:

$$
U-u_{n}\left(D, O^{\prime}\right)
$$

n'est plus discontinue en $O^{\prime}$ et comme en dehors de $O^{\prime}$ le potentiel $U$ n'admet que des masses positives, et $u_{n}$ que des masses négatives, la différence $U-u_{n}$ ne peut avoir que des maxima, et pas des minima; elle est d'ailleurs positive pour tous les points qui n'appartiennent pas aux éléments qu'on a déjà balayés; car avant d'obtenir $u_{n}$ en ces points on a

$$
U>0, \quad u_{n}=0 .
$$

On a donc dans tout le domaine $D$

$$
U>u_{n}\left(D, O^{\prime}\right)
$$

ce qui veut dire que la suite des $u_{n}$, constamment croissante, tend vers une limite finie.

On aurait pu évidemment, avec quelques changements, raisonner de la même manière sans supposer que $L_{0}$ est une circonférence, mais cela n'a pas d'intérêt.

Il résulte de là par les raisonnements des $\$ 5,6,7,8$ que le domaine $D$ est représentable sur un cercle. 


\section{$\S$ 15. Cas où la fonction de Green n'existe pas pour $D$.}

Nous allons maintenant rechercher ce qui se passe quand la fonction $V$ est constamment égale à I. Reprenons la fonction de GREEN $u\left(D_{1}, 0\right)$ et supposons encore que $L_{0}$ est une circonférence concentrique à $C_{0}^{\prime}$; envisageons la dérivée

$$
\frac{d u\left(D_{1}, 0\right)}{d r}
$$

aux différents points de $L_{0}$; nous avons vu au $\S 12$ qu'elle s'y comporte réguièrement; posons:

$$
H=\int \frac{d u\left(D_{1}, 0\right)}{d r} d s
$$

Elle représente au facteur $-2 \pi$ près, les masses négatives que les balayages ont amenés sur $L$; la masse négative totale étant $-\mathrm{I}$, on a:

Supposons d'abord

$$
H \leqq 2 \pi \text {. }
$$

$$
H<2 \pi,
$$

je dis que dans ce cas $V$ n'est pas constamment égale à $\mathrm{I}$.

En effet envisageons le domaine $d_{n}$ limité par $L_{n}$ et $L_{0}$ et imaginons que $O$ fasse partie de ce domaine, ce qui arrivera pourvu que $n$ soit assez grand. Ecrivons simplement $u$ au lieu de $u\left(D_{1}, 0\right)$ et $V_{n}$ au lieu de $V\left(d_{n}\right)$; nous aurons. la formule de Grene :

$$
\int\left(u \frac{d V_{n}}{d \nu}-V_{n} \frac{d u}{d \nu}\right) d s=2 \pi V_{n}^{0}
$$

Dans cette formule $V_{n}^{0}$ est la valeur de $V_{n}$ en $O$; l'intégration est étendue à tous les éléments $d s$ de la frontière d'un domaine contenant $O$; les dérivées $\frac{d}{d \nu}$ représentent les dérivées estimées suivant la normale à cette frontière, cette normale étant dirigée vers l'extérieur.

Nous pouvons appliquer cette formule au domaine $d_{n}$ dont la frontière se compose d'un nombre fini d'arcs de cercle, de sorte que toutes nos fonctions s'y comportent régulièrement.

Sur $L_{0}$ on a:

$$
\begin{array}{r}
u=0, V_{n}=\mathrm{I}, \frac{d u}{d \nu}=-\frac{d u}{d r} \\
\int_{L_{0}}\left(u \frac{d V_{n}}{d \nu}-V_{n} \frac{d u}{d \nu}\right) d s=H .
\end{array}
$$

Acta mathematica. 31. Imprimé le 21 mars 1907. 
Sur $L_{n}$ on a:

$$
\begin{aligned}
& V_{n}=0, u>0, \frac{d V_{n}}{d \nu}<0 \\
& \int_{L_{n}}\left(u \frac{d V_{n}}{d \nu}-V_{n} \frac{d u}{d \nu}\right) d s<0
\end{aligned}
$$

On a donc

$$
\int_{L_{n}}+\int_{L_{0}}=2 \pi V_{n}^{0} ; \int_{L_{n}}<0, \int_{L_{0}}^{0}=H
$$

d'où :

on a donc à la limite

$$
2 \pi V_{n}^{0}<H
$$

$$
2 \pi V^{0} \leqq H,
$$

$V^{\circ}$ étant la valeur de $V$ au point $O$, et si $H<2 \pi$

$$
V^{0}<\mathbf{I}
$$

La fonction $V$ n'est donc pas constamment égale à I, ce qui permet d'appliquer les démonstrations des deux $§$ précédents et de conclure que $D$ est représentable sur un cercle.

Supposons maintenant $H=2 \pi$.

Dans ce cas, je dis que le domaine $D_{1}$ est représentable sur un cercle.

En effet continuons à écrire $u$ au lieu de $u\left(D_{1}\right.$, o) et déduisons-en les fonctions $v$ et $z$ comme au $\S 5$. Bien que le domaine $D_{1}$ ne soit pas simplement connexe, la fonction $z$ sera uniforme, et en effet quand on tourne autour de $L_{0}$, $v$ augmente de $H$, c'est à dire de $2 \pi, z$ est multiplié par $e^{2 i \pi}$ et ne change pas.

A chaque point de $D_{1}$, correspond donc un point du plan des $z$ et ce point est situé à l'intérieur du cercle $\mathrm{I}$ de centre $O$ et de rayon $I$. Il faut voir maintenant si la fonction $z$ ne peut pas reprendre plusieurs fois la même valeur.

Reprenons les notations du $\$ 13$ en posant:

$$
\begin{gathered}
K_{n}=-\int \frac{d V\left(d_{n}\right)}{d r} d s, \quad K=-\int \frac{d V}{d r} d s, \\
W=\frac{2 \pi V}{K}, \quad W_{n}=\frac{2 \pi V\left(d_{n}\right)}{K_{n}}, \\
Z=e^{-\left(W+i W^{\prime}\right)}, \quad Z_{n}=e^{-\left(W_{n}+i W_{n}^{\prime}\right)},
\end{gathered}
$$

$W^{\prime}$ et $W_{n}^{\prime}$ ayant même sens que dans ce $\$$ I3.

Les conclusions précédentes subsisteront, c'est à dire que la variable $Z_{n}$ donnera la représentation conforme du domaine $d_{n}$ sur un anneau circulaire, 
compris entre les deux circonférences concentriques de rayon I et $e^{-\frac{2 \pi}{K_{n}}}$. Seulement dans le cas qui nous occupe, $K_{n}$ qui va constamment en décroissant, tend vers zéro, et on a $K=0, V=\mathrm{I}, W=\infty$. Je dis que $K_{n}$ va constamment en décroissant avec $n$; et en effet $V\left(d_{n}\right)$ va constamment eu croissant, sauf sur $L_{0}$ où elle reste égale à $\mathrm{I}$; il en résulte que sur $L_{0}$

$$
-\frac{d\left(V d_{n}\right)}{d r}
$$

et par conséquent $K_{n}$ vont en décroissant.

Cela posé, considérons $u\left(d_{n}\right.$, o) comme fonction de $Z_{n}$. C'est une fonction harmonique dans l'anneau circulaire $\left(\mathrm{I}, e^{-\frac{2 \pi}{K_{n}}}\right)$, sauf au point correspondant à $\circ$ où elle devient logarithmiquement infinie, et qui s'annule sur les deux circonférences qui limitent cet anneau. C'est done le logarithme du module d'une fonction doublement periodique de $3^{\text {e }}$ espèce par rapport au logarithme de $Z_{n}$.

$\mathrm{Je}$ précise; considérons un système de fonctions doublement périodiques admettant pour périodes $2 i \pi$ et $\frac{4 \pi}{K_{n}}$, de sorte que si $F\left(\log Z_{n}\right)$ est l'une de ces fonctions on ait

$$
F\left(\log Z_{n}\right)=F\left(\log Z_{n}+2 i \pi\right)=F\left(\log Z_{n}+\frac{4 \pi}{K_{n}}\right) .
$$

Nous pourrions les considérer comme des fonctions de $Z_{n}$, mais il vaudra mieux poser

$$
Y_{n} Z_{n}=e^{-\frac{2 \pi}{K_{n}}}
$$

de telle façon que $\left|Y_{n}\right|=\mathrm{I}$ le long de $L_{0}$ et $\left|Y_{n}\right|=e^{-\frac{2 \pi}{K_{n}}}$ le long de $L_{n}$.

Si nous posons alors:

$$
F\left(\log Z_{n}\right)=\Phi\left(Y_{n}\right),
$$

nous voyons que $\Phi$ est une fonction uniforme de $Y_{n}$ telle que

$$
\Phi\left(Y_{n}\right)=\Phi\left(Y_{n} e^{\frac{4 \pi}{K_{n}}}\right) .
$$

Mais si nous posons

$$
u\left(d_{n}, \text { o }\right)=\log \left|\Omega\left(Y_{n}\right) Y_{n}^{\lambda}\right|
$$

$\Omega$ sera une fonction de $3^{\text {e }}$ espèce, c'est à dire que ce sera une fonction uniforme de $Y_{n}$ satisfaisant à la condition

$$
\Omega\left(Y_{n} e^{\frac{4 \pi}{K_{n}}}\right)=\alpha \Omega\left(Y_{n}\right), \quad \alpha e^{\frac{4 \lambda \pi}{K_{n}}}=\mathrm{I} .
$$


$\alpha$ et $\lambda$ sont des constantes. Si $Y_{n}^{0}$ est la valeur de $Y_{n}$ qui correspond au point $O$, valeur que nous pouvons toujours supposer réelle puisque l'argument de $Y_{n}$ n'est déterminé qu'à une constante près, la fonction $\Omega$ devient infinie pour

et nulle pour

$$
Y_{n}=Y_{n}^{0}, Y_{n}=Y_{n}^{0} e^{\frac{4 m \pi}{K_{n}}} \quad(m \text { entier positif ou négatif })
$$

$$
Y_{n}=\frac{\mathrm{I}}{Y_{n}^{0}}, Y_{n}=\frac{\mathrm{I}}{Y_{n}^{0}} e^{\frac{4 m \pi}{K_{n}}}
$$

Elle se réduit à $\mathrm{I}$, pour $Y_{n}=\mathrm{I}$, et elle est par là entièrement déterminée par le quotient de deux fonctions $\theta$.

Il est aisé de voir que si nous posons:

$$
H_{n}=-\int_{L_{0}} \frac{d u\left(d_{n}, 0\right)}{d r} d s
$$

on aura $H_{n}<2 \pi, 2 \pi \lambda=H-H_{n}=2 \pi-H_{n}$.

On aura d'ailleurs:

$$
\log \left|Y_{n}\right|=-2 \pi \frac{1-V\left(d_{n}\right)}{K_{n}} .
$$

Lorsque $n$ croît indéfiniment, I $-V\left(d_{n}\right)$ et $K_{n}$ tendent vers zéro, mais leur rapport reste limité; je ne sais pas encore s'il tend vers une limite finie.

En effet la fonction harmonique

$$
V\left(d_{n+1}\right)-V\left(d_{n}\right)
$$

est constamment positive, et elle s'annule sur $L_{0}$; nous pouvons done lui appliquer le théorème de HARNACK, ce qui nous fournira une limite supérieure et inférieure du rapport

$$
\frac{V\left(d_{n+1}\right)-V\left(d_{n}\right)}{\bar{K}_{n}-\bar{K}_{n+1}}
$$

Ces limites s'appliqueront au rapport:

$$
\frac{V\left(d_{n+p}\right)-V\left(d_{n}\right)}{\bar{K}_{n}-K_{n+p}}
$$

ou en faisant croître $p$ indéfiniment, au rapport

$$
\frac{\mathrm{I}-V\left(d_{n}\right)}{K_{n}} \text {. }
$$

Ainsi en chaque point du domaine $D_{1}$, nous pouvons assigner une limite supérieure à

$$
\log \left|\frac{1}{Y_{n}}\right|
$$


Nous pourrions de même assigner une limite supérieure à ses dérivées: car si $V^{\prime}$ représente une des dérivées de $V$, le théorème de Harnack nous fournira encore une limite supérieure du rapport

Cela posé nous avons:

$$
\frac{V^{\prime}\left(d_{n+p}\right)-V^{\prime}\left(d_{n}\right)}{V\left(d_{n+p}\right)-V\left(d_{n}\right)}
$$

$$
\log |Q|=u\left(d_{n}, 0\right)+\lambda \log \left|\begin{array}{c}
\mathrm{I} \\
Y_{n}
\end{array}\right| .
$$

Je dis que quand $n$ croît indéfiniment, $\log |Q|$ tend uniformément vers $u\left(D_{1}, 0\right)$; en effet $u\left(d_{n}, 0\right)$ tendant uniformément vers $u\left(D_{1}, 0\right)$, on peut prendre $n$ assez grand pour que

$$
u\left(D_{1}, 0\right)-u\left(d_{n}, 0\right)<\frac{\varepsilon}{2} .
$$

De plus $\lambda$ tendant vers 0 , et $\log \left|\frac{\mathrm{I}}{\overline{Y_{n}}}\right|$ restant limité, on peut prendre $n$ assez grand pour que

d'où

$$
\lambda \log \left|\frac{\mathrm{I}}{\bar{Y}_{n}}\right|<\frac{\varepsilon}{2}
$$

$$
u\left(D_{1}, 0\right)-\log |\Omega|<\varepsilon .
$$

On démontrerait de même que les dérivées de $\log |\mathrm{Q}|$ tendent uniformément vers celles de $u\left(D_{1}, 0\right)$, puisque celles de $u\left(d_{n}, 0\right)$ tendent vers $u\left(D_{1}, 0\right)$ et que celles de $\log \left|\frac{\mathrm{I}}{Y_{n}}\right|$ sont limitées.

On en conclura que $\Omega$ tend uniformément vers $z$, et les dérivées de $Q$ vers celles de $z$ quand $n$ croît indéfiniment.

On verrait que dans le domaine $d_{n}$ la fonction $\Omega$ peut prendre la même valeur deux fois au plus. $\mathrm{Si}$ alors l'on considère deux points $O$ et $M$ du domaine $D$, et les valeurs correspondantes $Y_{n}^{0}$ et $Y_{n}$ de $Y_{n}$; nous avons vu que $\log \left|\frac{\mathrm{I}}{\overline{Y_{n}^{0}}}\right|$ et $\log \left|\frac{\mathrm{I}}{\bar{Y}_{n}}\right|$ sont limités; nous pouvons done assigner une limite inférieure à $\left|Y_{n}^{0}\right|$ et à $\left|Y_{n}\right|$. Soit alors $M^{\prime}$ un point du domaine $d_{n}$ pour lequel $\Omega$ reprenne la même valeur qu'au point $M$, et $Y_{n}^{\prime}$ la valeur correspondante de $Y_{n}$. On démontrerait, en se servant de l'expression de $\boldsymbol{\Omega}$ par les séries $\Theta$, que quand $n$ tend vers l'infini, et par conséquent $K_{n}$ vers zéro, ( $Y_{n}$ et $Y_{n}^{0}$ restant au dessus d'une certaine limite) $Y_{n}^{\prime}$ tend vers zéro, de sorte que le point $M^{\prime}$ s'éloignera indéfiniment sur le domaine $D_{1}$, il ne pourra par exemple rester dans le domaine $d_{p}$, où $p$ est un nombre fixe plus petit que $n$. On peut donc prendre $n$ assez grand 
pour que l'on soit assuré que $\Omega\left(Y_{n}\right)$ ne prendra pas plus d'une fois la même valeur dans le domaine $d_{p}$; mais nous avons vu que $\Omega\left(Y_{n}\right)$ tend uniformément vers $z$ quand $n$ croît indéfiniment (et qu'il en est de même pour les dérivées) donc en répétant le raisonnement du $\$ \mathrm{I}_{3}$, on verrait que z ne peut prendre qu'une seule fois la même valeur dans le domaine $d_{p}$ et par conséquent dans le domaine $D_{1}$.

Ainsi soit $C$ le cercle de centre $O$ et de rayon I; la fonction $z$ nous donnera la représentation conforme du domaine $D_{1}$ sur ce cercle, de telle façon qu'à tout point du domaine corresponde un point du cercle et un seul, et qu'à tout point du cercle ne puisse correspondre plus d'un point du domaine; à un point de $L_{0}$ correspondra un point de la circonférence du cercle et réciproquement.

Nous serons ainsi conduits à répartir les points du cercle $C$ en deux ensembles, l'ensemble $E$ des points auxquels correspond un point du domaine, (ensemble dans lequel nous ferons entrer la circonférence dont les points correspondent à ceux de $L_{0}$ ) et l'ensemble $E_{1}$ des points auxquels ne correspond aucun point du domaine.

Tous les points de $E_{1}$ sont intérieurs à une circonférence, intérieure ellemême à la circonférence de rayon 1 . Les points de la frontière de $E$, sauf ceux de la circonférence de rayon I, font partie de $E_{1}$. La domaine $E$ est doublement connexe, comme $D_{1}$ dont il est l'image. Si donc nous considérons une courbe fermée tracée dans $E$, tous les points de $E_{1}$ seront intérieurs à cette courbe ou tous extérieurs.

Deux hypothèses sont donc possibles:

I $^{\circ}$. Ou bien $E_{1}$ se compose d'un seul point.

$2^{0}$. Ou bien $E_{1}$ en contient une infinité; car si $E_{1}$ contient plus d'un point, il ne peut admettre de points isolés.

Soient dans cette $2^{\text {de }}$ hypothèse $q$, $q_{1}$ et $q_{2}$ trois points de $E_{1}$, le premier sur la frontière de $E$. Construisons avec ces trois points les fonctions auxiliaires d'OSGOOD :

$$
\%(w), \quad t=\log \left|\frac{1}{w}\right| .
$$

Cette fonction $t$ est harmonique dans $E$ sauf en $O$ où elle devient logarithmiquement infinie; elle est partout positive et tend vers $O$ quand on se rapproche de $q$. La fonction $w$ est uniforme dans $E$, parce que toute courbe fermée tracée dans $E$, n'enveloppe aucun des points singuliers $q, q_{1}, q_{2}$ ou les enveloppe tous trois.

On a done $t>u\left(d_{n}\right.$, o) et par conséquent

$$
t>u\left(D_{1}, 0\right)
$$


ce qui montre que $u\left(D_{1}, 0\right)$ tend vers zéro quand on se rapproche du point $q$; on en conclurait comme au $\$ 8$ que le point $q$ doit se trouver sur la circonférence de rayon 1. Mais il n'en est pas ainsi puisque tous les points de $E_{1}$ sont intérieurs à cette eirconférence.

Nous sommes donc ramenés à la Ière hypothèse, d'après laquelle $E_{1}$ se réduit à un seul point.

Pour nous résumer, la fonction $z$ donne la représentation conforme de $D_{1}$ sur un cercle pointé.

Nous avons donc les deux domaines $D_{1}$ et $C_{0}^{\prime}$, qui ont une partie commune $\delta$, et représentables le $\mathrm{I}^{\text {er }}$ sur un cercle pointé, le second sur un cercle. Nous pouvons alors, en appliquant les procédés de M. Schwartz (Monatsberichte de l'Académie de Berlin, Octobre 1870 , page 792) conclure que le domaine total:

$$
D=D_{1}+C_{0}^{\prime}-\delta
$$

est représentable sur une sphère pointée.

En résumé, un domaine $D$ simplement connexe quelconque est susceptible de représentation conforme soit sur un cercle (\$ $\mathrm{I} 3$ et $\mathrm{I} 4$ ) soit une sphère pointée (\$ $\left.\mathrm{I}_{5}\right)$.

Si alors nous nous reportons à la fin du $\S \mathrm{I} 2$, nous verrons que le $1^{\text {er }}$ cas ne peut être que celui où l'arc $B^{\prime} A^{\prime}$ ne se réduit pas à un point et le $2^{\mathrm{d}}$ cas celui où cet arc se réduit à un point. On peut donc donner au critère qui permet de discerner les deux cas plusieurs formes entièrement différentes les unes des autres et différentes aussi de celle qu'avait adoptée M. BRODín.

Revenons pour terminer à notre fonction $Y_{n}$; nous avons vu que nous pouvions lui assigner une limite supérieure et une limite inférieure; mais il pourrait encore se faire qu'elle oscille entre ces limites quand $n$ croît indéfiniment au lieu de tendre vers une limite déterminée. Les considérations précédentes montreraient qu'elle tend effectivement vers une limite déterminée qui est:

$$
\frac{z-z_{0}}{z-z_{0}^{\prime}} \frac{\mathrm{I}-z_{0}^{\prime}}{\mathrm{I}-z_{0}}
$$

$z_{0}$ étant l'affixe du point unique auquel se réduit l'ensemble $E_{1}$, tandis que $z_{0}^{\prime}$ est le point inverse de $z_{0}$ par rapport au cercle $C$. 